\title{
A Prototype Decision Aid for Evaluating and Selecting R\&D Proposals
}

R. A. Al Ayat

A. Lamont

A. Sicherman

May 1992

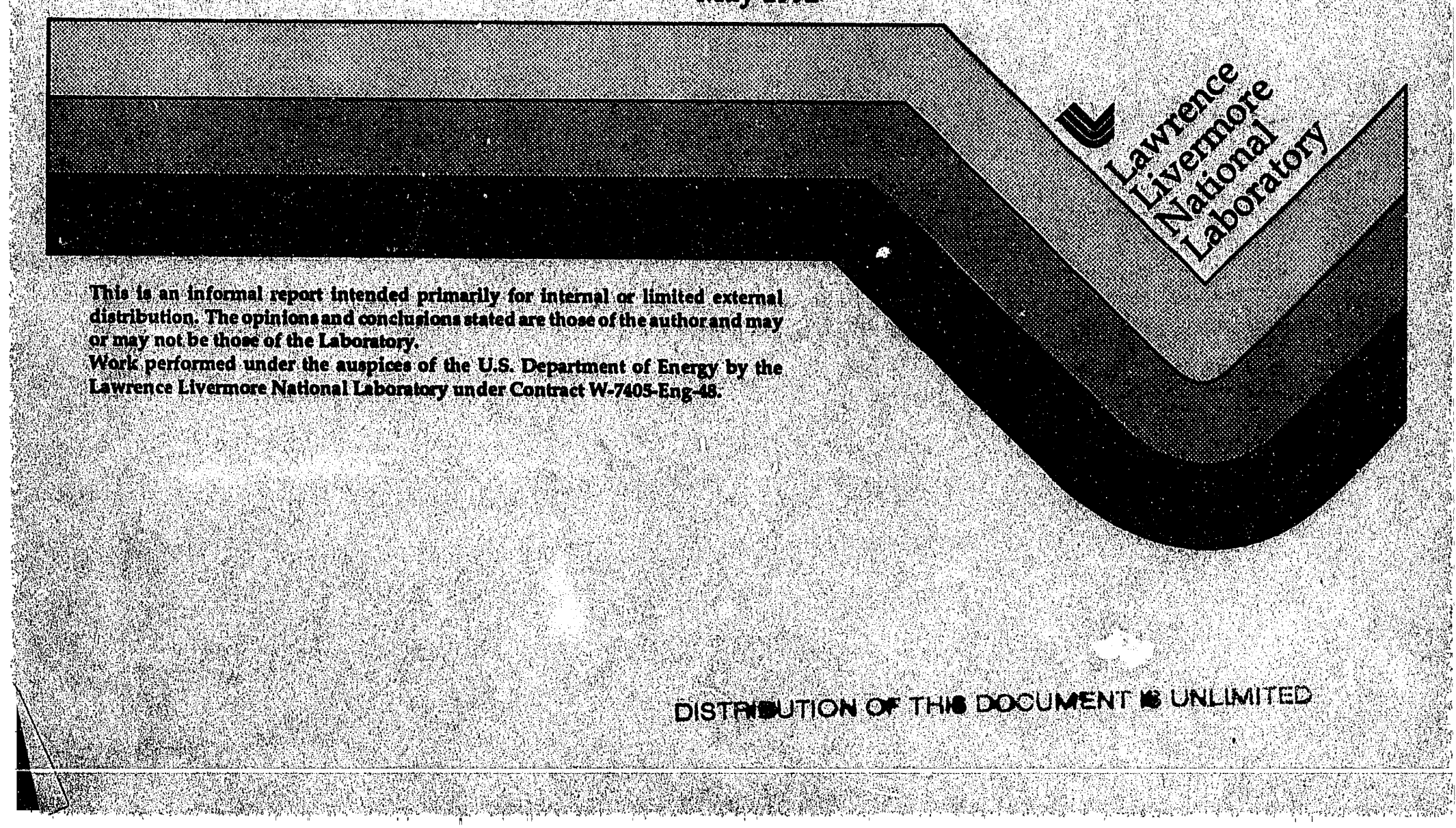




\section{DISELAMIER}

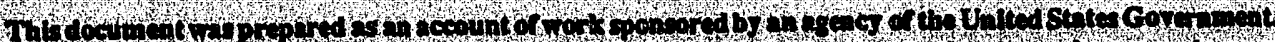

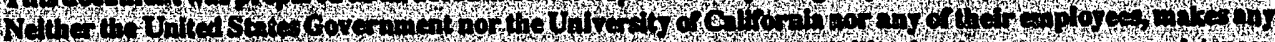

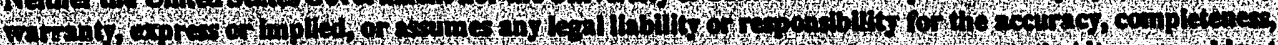

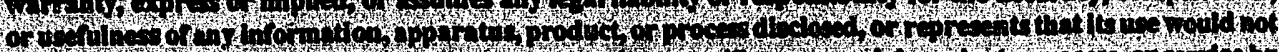

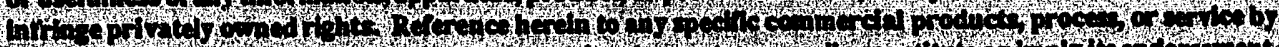

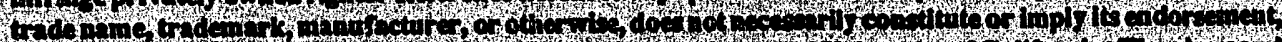
158

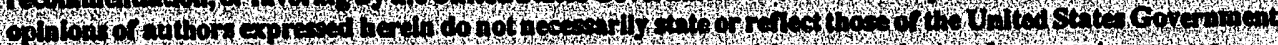

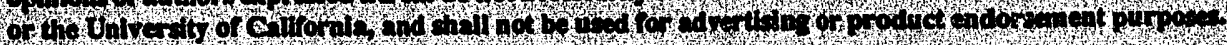

This reporthes bet rep od diced

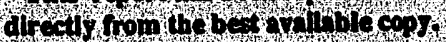

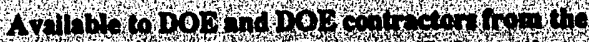

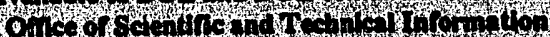

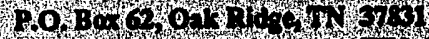

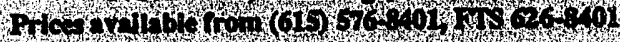

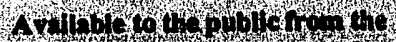

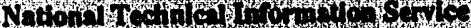

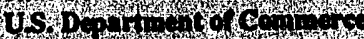

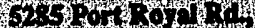
Springith V V 210 


\section{A Prototype Decision Aid for Evaluating and Selecting R\&D Proposals}

R. A. Al-Ayat

A. Lamont

A. Sicherman

Systems Research Group

Lawrence Livermore National Laboratory

Submitted to the Institutional Research and Development Committee

December 16, 1988

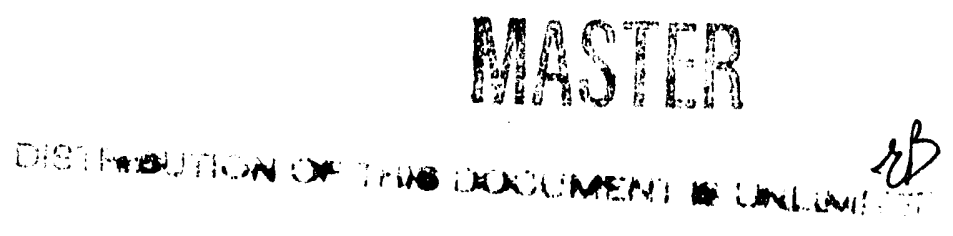




\section{A Prototype Decision Aid for Evaluating and Selecting R\&D Proposals}

\section{Introduction}

This report describes a prototype decision aid which has been developed to assist the Institutional Research and Development (IR\&D) Committee in selecting proposals for funding. This tool was requested to help address the following concerns about the IR\&D proposal selection process:

- Some good proposals might be overlooked simply because no one on the Committee advocates them forcefully.

- The process takes a lot of time.

- The final portfolio of proposals selected may not maximize the long-run benefits to the Laboratory.

These concerns stem from the observation that there is no formal framework for making distinctions between proposals, or weighing and comparing those distinctions. It was felt that the process could be improved by a framework that:

- Provides explicit descriptors that Committee members can use to evaluate and compare different features of proposals.

- Encourages the Committee to use a uniform, systematic scheme for evaluating the proposals.

- Helps the Committee focus more quickly on the issues that are truly relevant for distinguishing between proposals.

In March of this year, at the Committee's request, we proposed a sequence of six tasks for developing a decision aid which addresses these concerns. In our scope of work for FY88, we performed three tasks:

Task 1. Developed a systematic framework for describing the merits of individual proposals. In this task, we worked with committee members and attended several committee FY89 proposal evaluation meetings to identify key factors characterizing the significance of a research proposal and its potential for success. We developed a checklist of attributes and 
measurement scales which describes how well proposals address key factors and thus provide a basis for systematically comparing proposals.

Task 2. Developed an analytic form for a ranking function which can be used to compare proposals. This ranking or utility function synthesizes the scores that proposals receive on the measurement scales from Task 1 and produces an overall figure of merit for each proposal. The analytic form is to be calibrated based on preference assessments reflecting the value judgments of committee members. The functional form is designed so that different types of preference viewpoints can be readily and consistently quantified.

Task 3. Developed prototype software to store data about proposals, perform the required analysis, and produce tables of proposal features and rankings.

Tasks 1-3 provide a basic tool for systematically describing, displaying, and rankordering proposals. Tasks 4-6 dealing with portfolio concerns (i.e., features of a group of proposals such as an appropriate mixture of low and high risk proposals) are discussed later as possible future work.

Our report is organized as follows. We first discuss how attributes and measurement scales are used to describe proposals and how a utility function can be used to rank-order proposals. We then describe the prototype software. We conclude with a discussion of future work. For simplicity of presentation, much of the technical detail is relegated to four appendices. Appendix A contains the definitions of the attributes and measurement scales. Appendix $B$ describes the ranking function. Appendix $C$ summarizes features of the prototype software. Appendix $D$ contains our March proposal, including tasks for possible future work.

\section{Approach to Evaluating and Comparing Proposals}

Figure 1 shows the steps followed in using the decision aid to help evaluate proposals. These steps are basically similar to the activities already followed by the Committee, but here they have been given more structure. We give a quick overview of these steps and then discuss selected points in more detail.

In the first step, proposals are described using a set of well-defined measurement scales called attributes. These attributes address such issues as the technical quality of the proposed approach, and the nature of the impact the research might have, if it is successful. Each proposal is reviewed by members of the Committee and is assigned an appropriate level on each measurement scale. The attribute descriptions are intended to be objective in the sense that no value judgements are made at this point about the relative importance or significance of the attributes. Every person may have a somewhat different opinion about the relative importance of different attributes. Such preferences are addressed in the third step discussed below.

Once each proposal has been described using the scales, those which tend to score poorly on most, or all, of the attributes can be screened out and set aside. This 


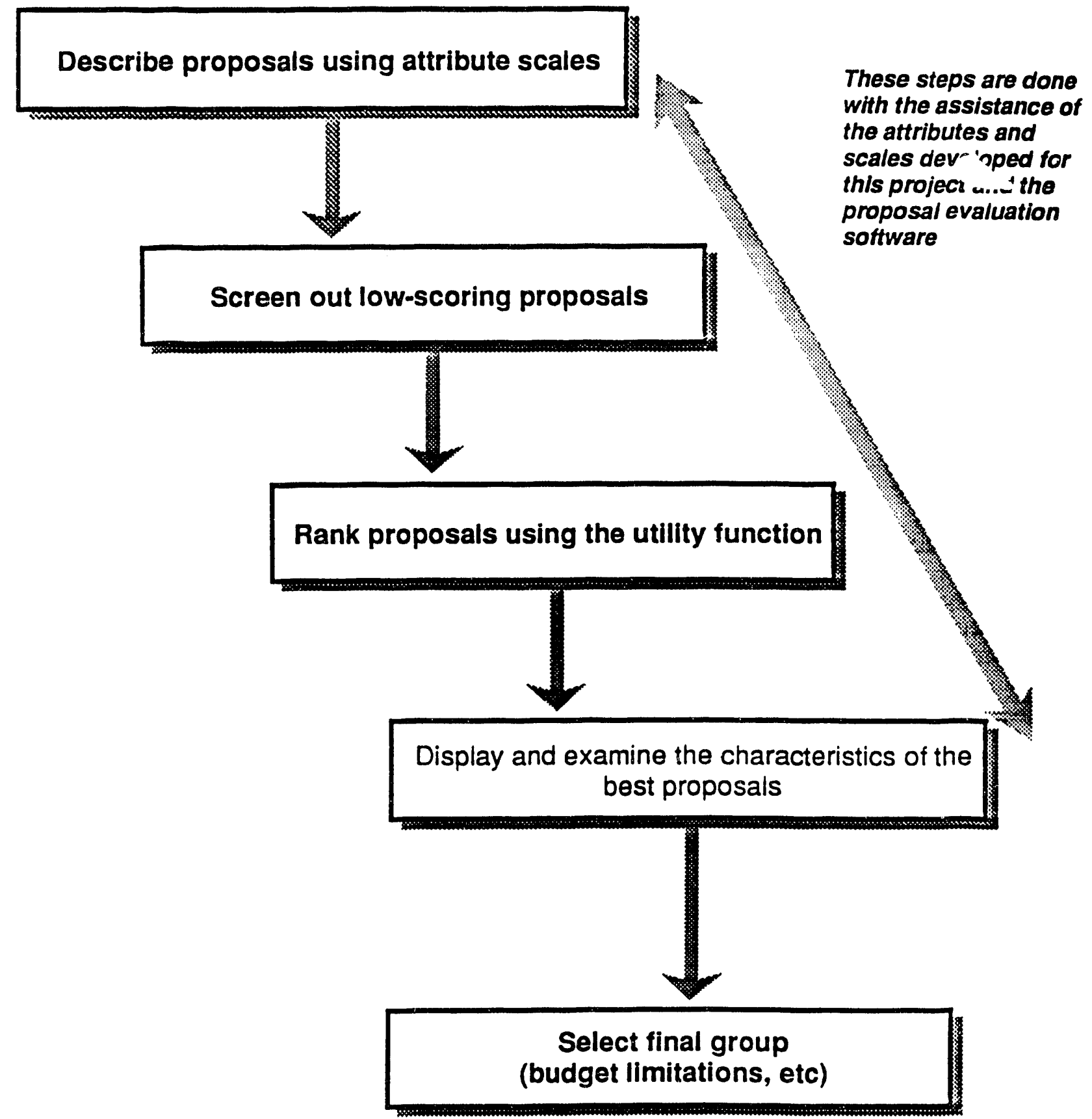

Figure 1. The decision aid developed here will assist the Committee in evaluating and ranking proposals. 
screening is done in the second step of Fig. 1. The attribute definitions allow such screening to be formally described and documented.

In the third step, the proposals are compared. Although the attribute levels of the proposals have been specified at this point, value judgements are needed in order to compare one proposal to another. A utility function (i.e., a ranking or preference function) is used to compute the utility or figure of merit of each of the proposals. This utility function is calibrated so that its parameters take a preference viewpoint into account. The utility function takes a proposal's attribute levels as input and produces the proposal's overall utility, or ranking score, as output.

It is quite possible to have several different utility functions, each one reflecting a different point of view. Of course, the rankings of the proposals might be different for each different utility function. In addition, preferences may change from year-to-year, reflecting changes in research policy. For example, the relative importance of concerns like "programmatic impact" versus "positioning of the Lab" for different kinds of future activities may change over time. Thus, different and changing preference viewpoints can be reflected by different calibrations of the ranking function.

In the fourth step, given the proposal ranking, the Committee can examine those at the top of the list. Generally the Committee will recommend for funding as many of the high-ranking proposals as the budget allows. At this stage some additional issues may be taken into account. There are often questions about whether or not a particular proposal should be funded by IR\&D or by an existing program. There may also be some consideration of the diversity of the highest ranking proposals. For example, the Committee might wish to fund a few proposals that have somewhat higher risks but promise larger long-term payoffs to the Laboratory. The prototype software that has been developed can assist in some of these deliberations by identifying proposals that score well on attributes such as long-term return to the Laboratory.

Because of the decision aid's formal structure, the committee can readily examine the sensitivity of ranking results to various assumptions made and preference viewpoints used. If the rankings change significantly, debate can be focused on the critical issue requiring consensus.

The fifth step in Fig. 1 refers to the recommendation by the Committee of the final group. In this step, the Committee considers where the cutoff in recommended proposals occurs due to budget limitations, and which "borderline" proposals should be included if the funding were available. This siop is separated from the others in Fig. 1 to reemphasize another point: the decision aid is designed to assist the Committee and does not replace in any way the decision making done by the committee.

The sections below further discuss two key aspects of the four steps shown in Fig. 1--namely describing proposals using attributes and quantifying value judgments using a utility function. 


\section{Assigning Attribute Levels of Proposals}

We have identified a set of 14 attributes or measures which cover features of a proposal that are important for judging its value. These attributes fall into four groups relating to key overall characteristics of a research project.

1. Likelihood that research will be successful.

2. Scientific significance of the research results.

3. Programmatic impact of the research.

4. Project's contribution to long-term positioning of the Lab.

We developed a measurement scale for each of the 14 attributes. Figure 2 shows the relationship of the 14 attribute scales (S1, S2, etc.) to the 4 major proposal characteristics listed above. A more complete description of these groups, attributes, and scale definitions is included in Appendix A. As an example, the attributes related to scientific significance are discussed here.

The scientific significance of a proposal is described by three attributes (S8, S9, and $\mathrm{S} 10$ in Fig. 2):

1. The creativity and degree of innovation of the proposed research.

2. The recognition for the Laboratory that would result if it is successful.

3. The usefulness of the results.

For each of these attributes we have established a scale for describing the proposals. For example, the scale for the "creative/innovative" attribute has four levels:

Level 4: The research will result in a unique or novel reaction, material, experimental device, model, or algorithm; it studies phenomena not focused on before; level of research is ahead of others or unique.

Level 3: A novel application to one area of theory or tools developed in another area; novel synthesis or combination of technologies; simplification of current complex approach; novel experimental setup; level of research ahead of others.

Level 2: Extends current tools in existing areas; proves out new tools others have developed; level of research ahead of others.

Level 1: Level of research equal to what others are doing.

Similar scales have been developed for the other thirteen attributes.

When a reviewer evaluates a proposal, he or she "scores" the proposal by deciding which description (e.g., scale point) best fits the proposal. The reviewer scores the 


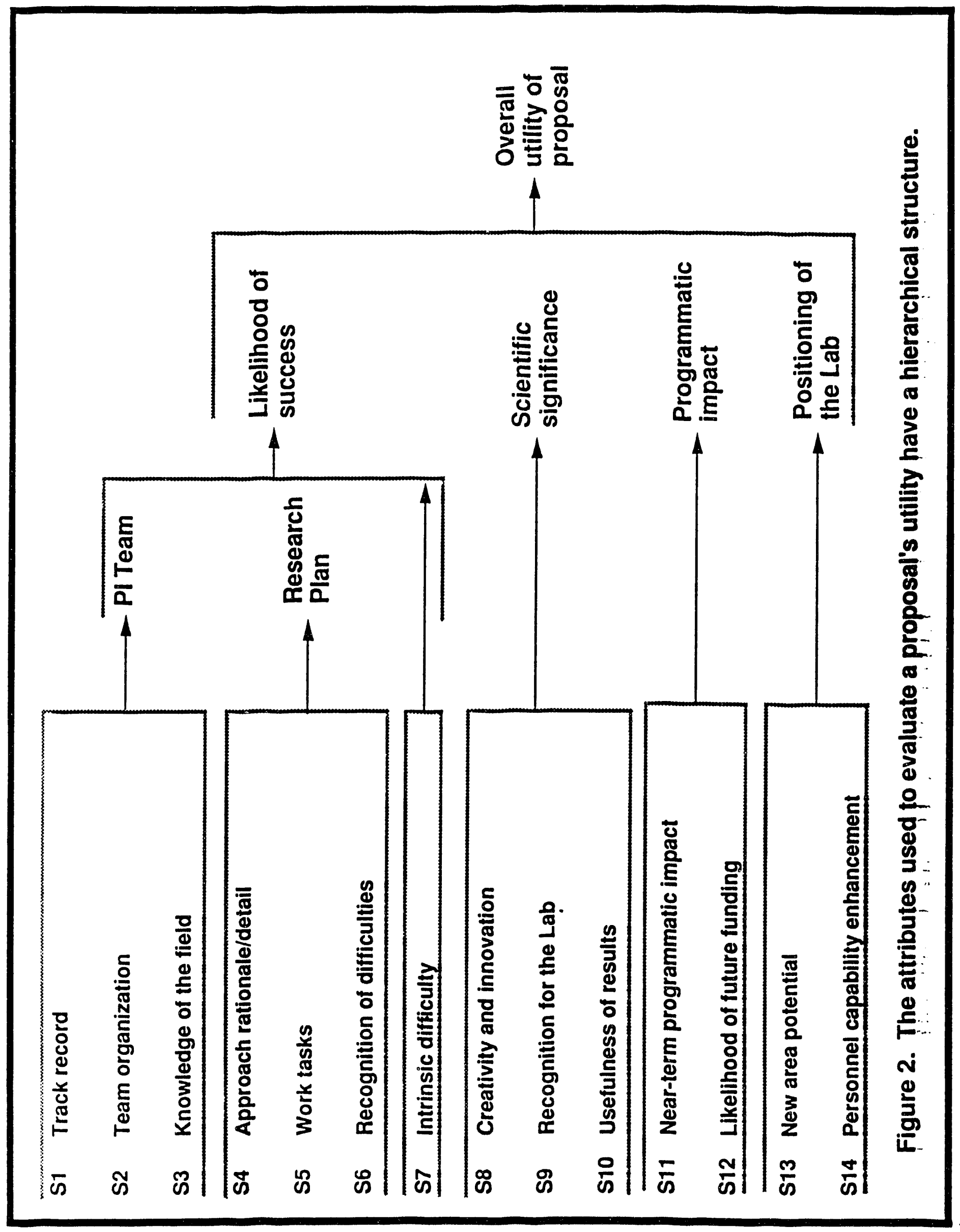


proposal for all the attributes listed in Appendix A. Figure 3 shows an example of a form that Committee members could use to record the scores given for each proposal.

Note that two reviewers may not exactly agree on the best description for a proposal. One person might feel that the proposal will result in a truly novel method, while another might look at the same proposal and feel that it is better described as a novel application of methods developed in one area to a problem in another area. Nonetheless, this argument centers around relatively objective issues. They are not arguing about how significant this attribute is compared to other attributes, and they are not asked to make any judgments about the relative value of the different points on this scale. By separating the issue of describing the features of a proposal from the issue of how important a given feature is, we hope that the Committee's discussions can be more clearly focused.

\section{Comparing the Proposals Using a Utility Function}

Comparing proposals requires judgments about the relative values of the points on each attribute scale and about the relative importance of the attributes. These judgments are quantified by the utility function. The utility function is calibrated through an interview with the person(s) whose judgments are to be represented. It is essentially a model of the way that the person makes choices between alternatives.

To model performance judgments effectively and consistently, one must be careful not to make ad hoc assumptions that may be inappropriate. For example, we do not simply "add up" the attribute scores to get an overall score. To illustrate heuristically why not, we refer to the 4 levels of the scale for the creative/innovative attribute. Just because there are 4 levels it does not follow that level 3 is two/thirds of the value between level 1 and level 4. Depending on the preference viewpoint, the value could be more or less. Similarly, it does not follow that all the level 4's on separate scales are of equal importance, as would be implied if they are simply added.

Finally, it may not be appropriate to combine attribute values with an "aciditive" model. This is especially true if having an excellent score on one scale compensates dramatically for having a poor score on another scale, or if having a poor score on one scale negates almost completely a good score on another scale. An additive model cannot reflect these kinds of preference viewpoints. The model form we have selected is flexible enough to reflect a much wider range of possible viewpoints. Appendix $B$ discusses utility functions in general and the particular form of the utility function developed for this decision aid.

Because there is no unique correct set of value judgments, there can be as many different rankings as there are members of the Committee, even if all members agree on the basic descriptions (i.e., attribute level assignments) of the proposals. It is expected here that the Committee would use one primary set of judgments (i.e., one primary utility function) for a base-case ranking. One or two auxiliary utility functions could also be developed which would represent other points of view. For example, one utility function might place a great deal of importance on long-term positioning of the Lab, while another might place more importance on high likelihood of success and strong programmatic impact. It is quite possible that many of the proposals that are -6- 
Proposal:

Evaluator:

\begin{tabular}{|r|r|c|c|}
\hline \multicolumn{2}{|c|}{ Evaluation Criteria } & $\begin{array}{c}\text { Range } \\
\text { Worst-Best }\end{array}$ & $\begin{array}{c}\text { Proposal } \\
\text { Rating }\end{array}$ \\
\hline \multicolumn{2}{|c|}{ LIKELIHOOD OF SUCCESS } \\
PI TEAM
\end{tabular}

See description sheets for scale-point definitions

Note: The rating assigned should correspond to the scale point rating definition in the evaluator's judgment. The evaluator may interpolate if desired (e.g., assign a 2.5 if better than a 2 but not quite as good as a 3, given the scale point definitions).

Figure 3. Example form for recording the reviewer's evaluation of a proposal's attributes. 
favored under one utility function will continue to be favored under the other. In these cases, the Committee can be quite confident that these are indeed strong proposals.

The utility function provides an overall rating of each proposal. In addition, the utility function is structured so that comparisons can be made with a focus specifically on the characteristics of likelihood of success, scientific significance, programmatic impact, and positioning of the Laboratory.

The decision aid is designed so that ranking results can be traced systematically to proposal scores on the evaluation criteria and to the Committee preference inputs. The Committee can readily examine the sensitivity of results to various assumptions made (e.g., attribute levels assigned) or preference viewpoints (e.g., relative importance of the four characteristics). If the rankings change significantly, the decision aid helps pinpoint the issues requiring consensus.

\section{The Prototype Proposal Evaluation Software}

The preceding approach to evaluating and comparing proposals has been implemented in a prototype software program on a personal computer. The program manages a data base which stores information about the proposals and the utility function used to evaluate them. This prototype is implemented in Foxbase on the IBM PC. We expect that the prototype can be made to run on the Macintosh relatively easily (Foxbase has a Macintosh version) to take advantage of the user interface there.

The existing prototype does not produce graphic displays of the output. This is largely due to constraints of time and budget. In a future version, it would be desirable to also include graphics.

There are a number of analysis options in the program which will evaluate the utilities of the proposals and then display the results in several ways. Appendix C describes the use of the program. The paragraphs below give an overview of the analyses that it can help perform.

To use the program, we first describe each of the proposals in terms of the attribute scales. Essentially, forms for each proposal (such as the one shown in Fig. 3) are entered into the data base. Next, the parameters of the utility function are entered. These parameters are assessed during an interview with the person(s) whose utility function is to be represented. Typically, a primary utility function is input as a "base case."

Once this information is entered the user can perform three kinds of analyses:

- Two-phase screening analysis. In the first phase, proposals that score in the low range on key attributes can be removed from consideration. The user determines which attributes are "key." For example, a proposal which has low scores on all the attributes for scientific significance may be screened out. In the second phase, the user can instruct the program to bring in proposals 
scoring well on particular attributes, even if they were screened out in the first phase. The two-phase screening creates a subset of proposals from the original group submitted. This subset is absent those proposals with significant drawbacks (first phase). Proposals with drawbacks that have special "plusses" are put back in the subset during the second phase. A subset of proposals can be more convenient to deal with in subsequent displays and other analyses. However, it is not mandatory to screen any proposals before ranking.

- Dominance analysis. This is a value-free method of identifying particularly good proposals. It is based strictly on the attribute levels of the proposals and does not use the utility function. It identifies those proposals that generally have higher attribute levels than all the other proposals. As an extreme example to illustrate the concept, a group of proposals each with attribute levels better than or equal to any proposals not in the group, will be the preferred subset regardless of viewpoint; i.e., they "dominate" the others.

- Utility analysis. The proposals are ranked using the utility function.

As already suggested, to streamline the analysis, one can define subsets or groups of proposals on which to focus. One useful group would be all the proposals submitted; another group might be all the proposals that have at least an acceptable score on every attribute. Another group might be, say, all the biomedical proposals. The software allows for convenient specification of such groups.

The results of these analyses are currently reported in tabular form. We anticipate adding graphic displays at a later time. The following types of displays are available now:

- Individual proposal. The display shows the proposal levels for all its attributes and a utility analysis showing overall utility and the utility values for the characteristics (a) likelihood that research will be successful, (b) scientific significance of the research results, (c) programmatic impact of the research, and (d) the project's effect on long-term positioning of the Laboratory.

- Utllity ranking of proposals in a group. The proposals can be ranked by overall utility and by the utility of each of the major characteristics. With such rankings, (e.g., by likelihood of success) the user can identify proposals that rank high on each characteristic.

- Detailed breakdown of utilities for a group of proposals. A table is printed showing the utilities of each proposal for every attribute. (See Appendix $\mathrm{B}$ for a discussion of individual attribute utilities and overall utility.)

- Dominance anaiysis results. For each proposal, the number of proposals 'hat it dominates and the number of proposals that dominate it are listed. One proposal dominates another if it has levels equal to or better than another for all the attributes.

Several of these ranking displays can also show the costs of each proposal and the cumulative costs as one goes down the rank-ordered list. These ranking displays 
and the dominance analysis can help identify the most desirable subset of proposals to fund from the entire list that was originally submitted.

\section{Summary and Future Work}

We have developed a prototype decision aid to assist the IR\&D Committee in selecting proposais for funding. With our approach, all proposals can be evaluated and ranked using a checklist of measuring scales (attributes), thereby:

- Reducing the chance of overlooking good proposals at the early stages of examination (because all important aspects of a proposal must by covered).

- Focusing on key comparative points of proposals and streamlining discussions away from peripheral technical details.

- Promoting fair and equitable treatment of all proposals since they are all evaluated on a set of common scales.

The really top proposals that score high on all the measures can be identified very quickly. Similarly, the rationale for screening those that score very low is formally provided. The decision aid can be used to supplement what the committee currently does, and the aid does not interfere with, or preclude, any other commitiee procedures or prerogatives.

Our FY88 effort was directed towards developing a prototype tool to illustrate the concepts and the feasibility of software implementation of a decision aid. We feel it is very desirable to iterate on and refine the results of this study to develop a final tool for future IR\&L proposal selections. Specifically, future work should include:

- Refining the attribute scales based on committee members' comments and illustrating level definitions with examples to reduce ambiguity.

- Refining the software interface, output displays, and implementation features.

- Refining and adding further information to evaluation forms.

- Assessing a base-case ranking function.

- Demonstrating the refined tool on last year's proposals.

Progressing from the current prototype to the final aid will require assistance from selected members of the IR\&D Committee. Specifically, we would want them to experiment with the existing prototype by evaluating previously submitted proposals. Once the current tool is refined, they can help us verify that:

1. The attributes that have been defined do adequately describe a proposal. 
2. The scales that have been defined for each attribute are operational and reviewers feel comfortable making assignments for real proposals.

3. The analyses produced by the decision aid are truly helpful in selecting proposals.

We also believe that the decision aid can be extended to help address additional concerns about selecting a portfolio of proposals. When the Committee views a portfolio of proposals together as a group, the members may have a perspective different from the one they use when considering each proposal separately. A simple example illustrating this point is that of almost redundant proposals submitted by two separate teams. Even if both proposals are of high quality, it makes little sense to fund both, and the Committee might typically fund the better of the two. Another example is that of risk "balance." Viewed in isolation, it may be hard to defend funding a high-risk proposal even though the payoff could be significant. When that proposal is part of a group, however, the portfolio as a whole may not be deemed risky. The Committee may desire a balance between high-risk/high-payoff and lower-risk/lower-payoff proposals in a final portfolio. Tasks 4-6 in Appendix D outline activities for extending the current decision aid to help address portfolio concerns such as balance and diversity. Portfolio issues can affect the maximization of long-run benefits to the Laboratory achieved by R\&D funding. 


\section{APPENDIX A}

\section{Descriptions of Attributes and Scales}

This appendix describes the attributes and scales which have been incorporated into the preliminary proposal evaluation aid. Although this set appears to capture most of the important features of proposals, we expect to refine these measuring scales after the Committee has experimented with the prototype software and applied it to some past proposals.

\section{The Four Primary Proposal Characteristics and Attribute Hierarchy}

The set of attributes has a hierarchical structure as shown in Fig. A-1. The overall utility of a proposal is determined by four primary characteristics. These are:

1. Likelihood of success.

2. Scientific significance.

3. Programmatic impact.

4. Positioning of the Laboratory.

These four characteristics can be decomposed into subconcerns, which in turn can be described using concrete scales or attributes. The scales are denoted by $\mathbf{S 1}$ through $\mathrm{S} 14$ in Fig. A-1. In such a hierarchy, we sometimes refer to a characteristic such as likelihood of success as a supra or vector attribute. A vector attribute has subattribites which in turn can also be vector attributes such as the PI Team in Fig. A-1. At the leftmost part of Fig. A-1, we have attributes that are no longer subdivided. These are called scalar attributes. Each has a measuring scale that can be used by the Committee members to describe a proposal in each area of concern. The overall rating of a proposal will be based on the rating for each attribute and the relative importance of the attributes. 
The sections below describe the attributes. Currently, there are fourteen scales for evaluating the scalar attributes. For simplicity of presentation, the proposed scales are not described in the text, but instead are shown in the figures directly below their corresponding subattributes in the hierarchy.

\section{Likelihood of Success}

The first major characteristic is the likelihood that the research team will accomplish their proposed tasks. The likelihood of success depends on:

1. Principal investigator $(P I) /$ team;

2. Research plan; and

3. Intrinsic difficulty.

For the Pl/team, we consider three subattributes: R\&D track record, the team makeup and organization, and the team's knowledge of the field. Similarly, to evaluate the research plan, we consider: the rationale and detail of the approach, whether or not the plan recognizes possible difficulties in conducting the proposed research, and whether or not the tasks are well planned.

To evaluate the likelihood of success, we must also consider the intrinsic difficulty of the proposed research. This reflects the degree to which the proposed goal is attainable given the best possible team, with all necessary resources.

Figure A-2 shows the general organization of the subattributes under likelihood of success. Figures A-3 through A-5 show the scales for rating each subattribute of the likelihood of success attribute.

\section{Scientific Significance}

The scientific significance attribute describes the creativity and innovation of the research, the usefulness of the results to the scientific community, and the recognition it would bring to the Laboratory. Conceivably, a proposal might be rated very high on this attribute even if it did not support any of the programs at the Laboratory. This attribute reflects the need for fostering high quality R\&D activities which will keep the Laboratory at the forefront of technology. This attribute can be divided into the following three subattributes:

1. Creativity and innovation;

2. Recognition for the Laboratory; and

3. Usefulness of results. 
Figure A-6 shows the organization of these subattributes and the proposed scales for rating them.

\section{Programmatic Impact}

This attribute describes the extent to which the research will, if successful, directly support recognized programmatic objectives or lead to increased funding in currently foreseeable areas. A proposal that can, after initial IR\&D funding, achieve independent programmatic support is more desirable both from the program and the IR\&D perspectives. Thus, for this attribute we suggest two subattributes:

1. Near-term programmatic impact; and

2. Likelihood of future funding.

These subattributes and their scales are shown in Fig. A-7.

\section{Laboratory Positioning}

Laboratory Positioning describes the extent to which the research will help the Laboratory move into new areas of research. It is different from the Programmatic Impact attribute in that it focuses on longer range, more speculative, and less welldefined opportunities. We propose using two subattributes. The first reflects the potential for developing a new area of research or a program after the initial IR\&Dfunded effort. The second subattribute describes the extent to which the research may enhance personnel capabilities at the Laboratory by maintaining state-of-the-art skills, attracting highly-respected scientists, or by contributing to a major career achievement for the $\mathrm{Pl}$ and team members. Figure A-8 displays the scales for these subattributes.

After committee member feedback and definition refinements, the attributes and scales should to the extent possible be:

- Complete: Include all areas of concern and be capable of indicating the degree to which overall concerns are met.

- Operational: Meaningful to the Committee members and facilitate explanations to others.

- Nonredundant: Avoid double-counting.

- Practical to use: Proposal evaluations can be made without taking an inordinate amount of time or effort.

- Well-defined: Attributes are unambiguous and do not lend themselves to varied interpretations by different evaluators. 
Table A-1 summarizes the focal points addressed by each scale discussed in this appendix. 
Table A-1. Summary of "focal points" or basic issues for each scale.

S1 Does track record of proposers show potential or demonstrated ability to carry out the research?

S2 Does team have the necessary people committed and appropriately organized?

S3 Does team have knowledge of the field and an awareness of the relevant activities of others?

S4 Are the problem and objectives clearly understood and stated and is there a well-delineated, judiciously selected plan of attack?

S5 Are the deliverables/milestones clearly described and appropriately supported by resource allocations and the funding requested?

S6 Are the challenges posed by the research goals understood and appreciated?

S7 How intrinsically feasible is the proposed research?

S8 Is the research unique; is the level of research ahead of others working in the field?

S9 Is the research perceived to be of board or narrow interest to the research community and nation?

S10 What big difference will the proposed work make in terms of usefulness of results to the scientific community?

S11 Will this work, if successful, lead to important and relevant impacts on current LLNL programs?

S12 Will this work, if successful, be funded by other sources in the future?

S13 Could this work lead to a new program or have broad impacts on Lab technical capabilities?

S14 Will this work enhance personnel capabilities? 


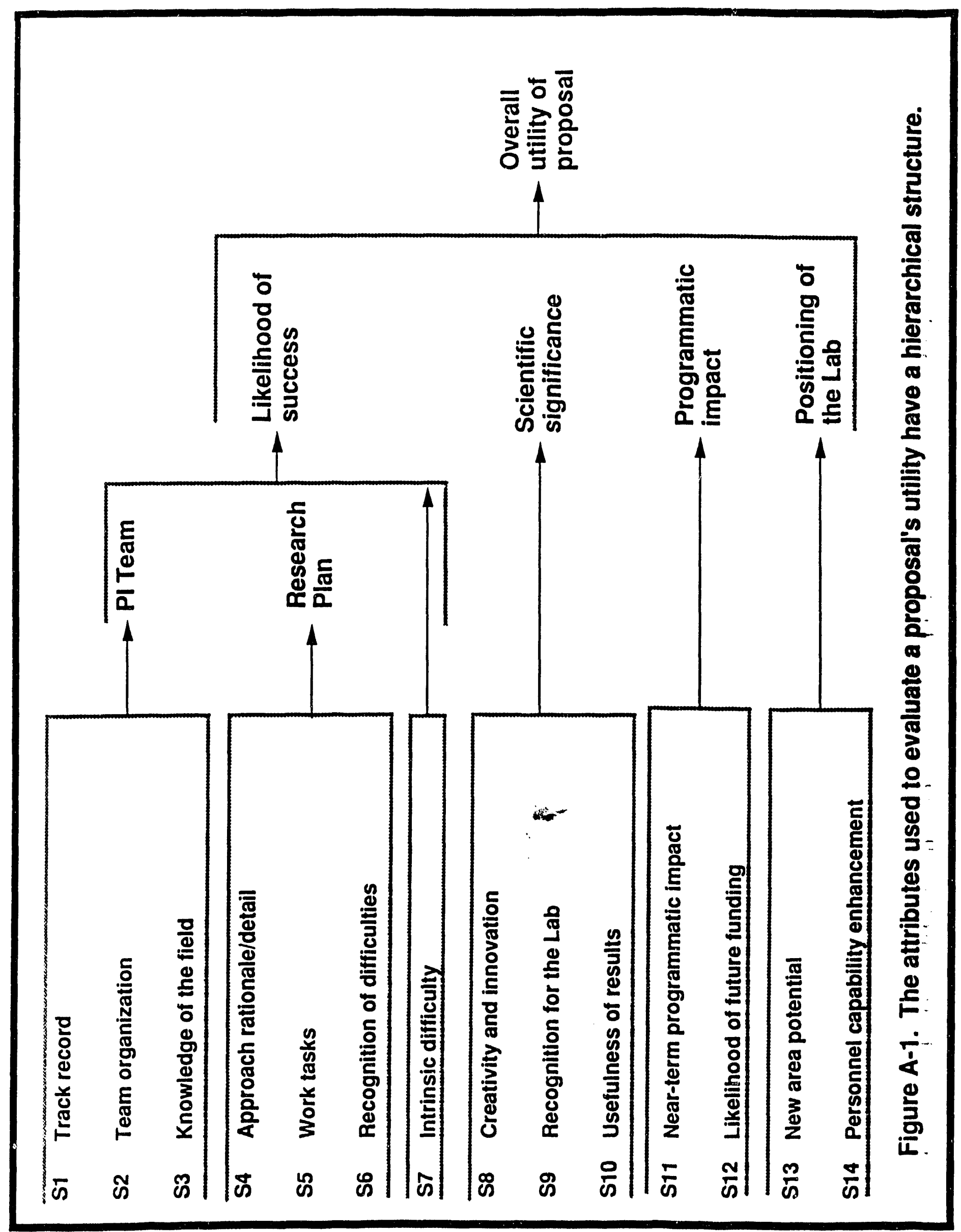




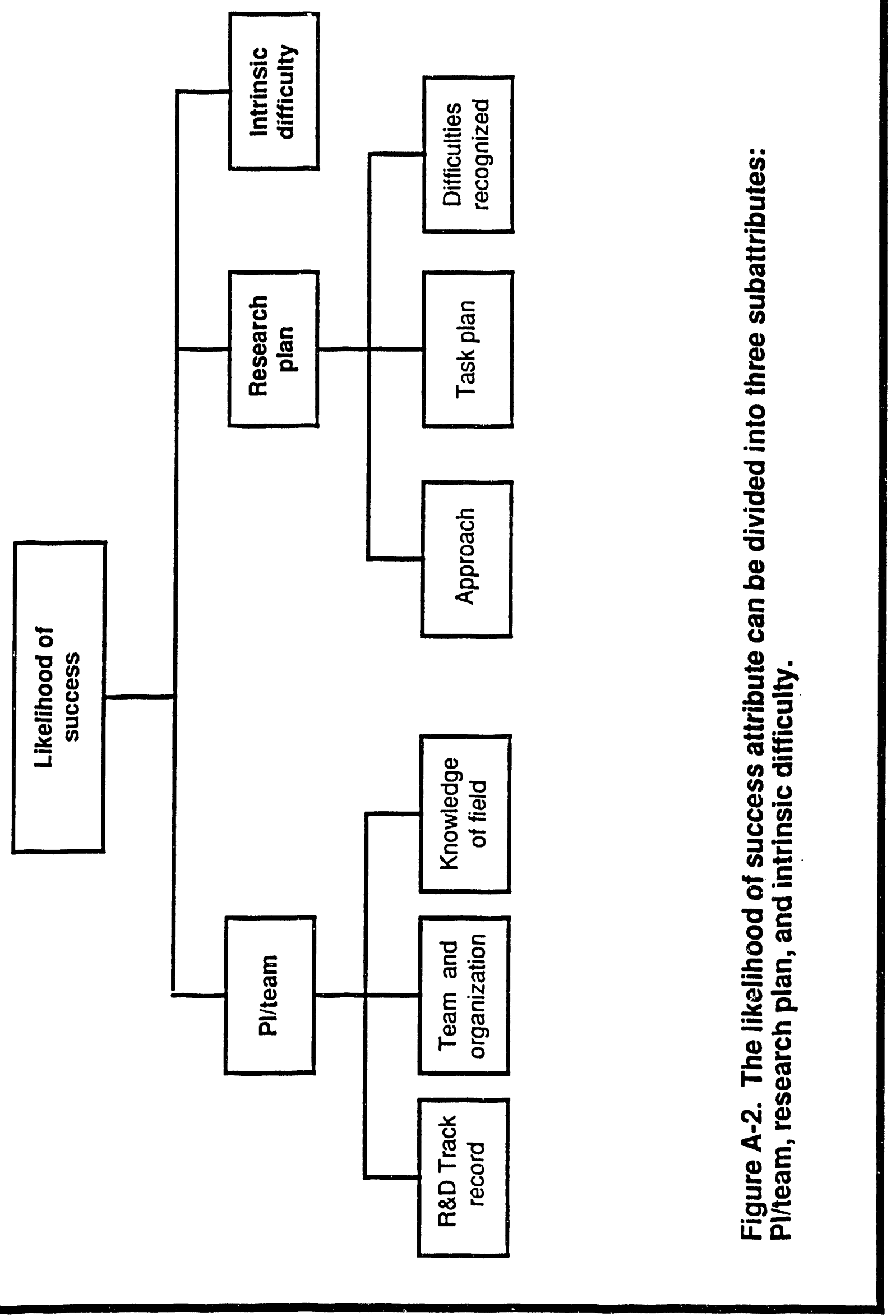




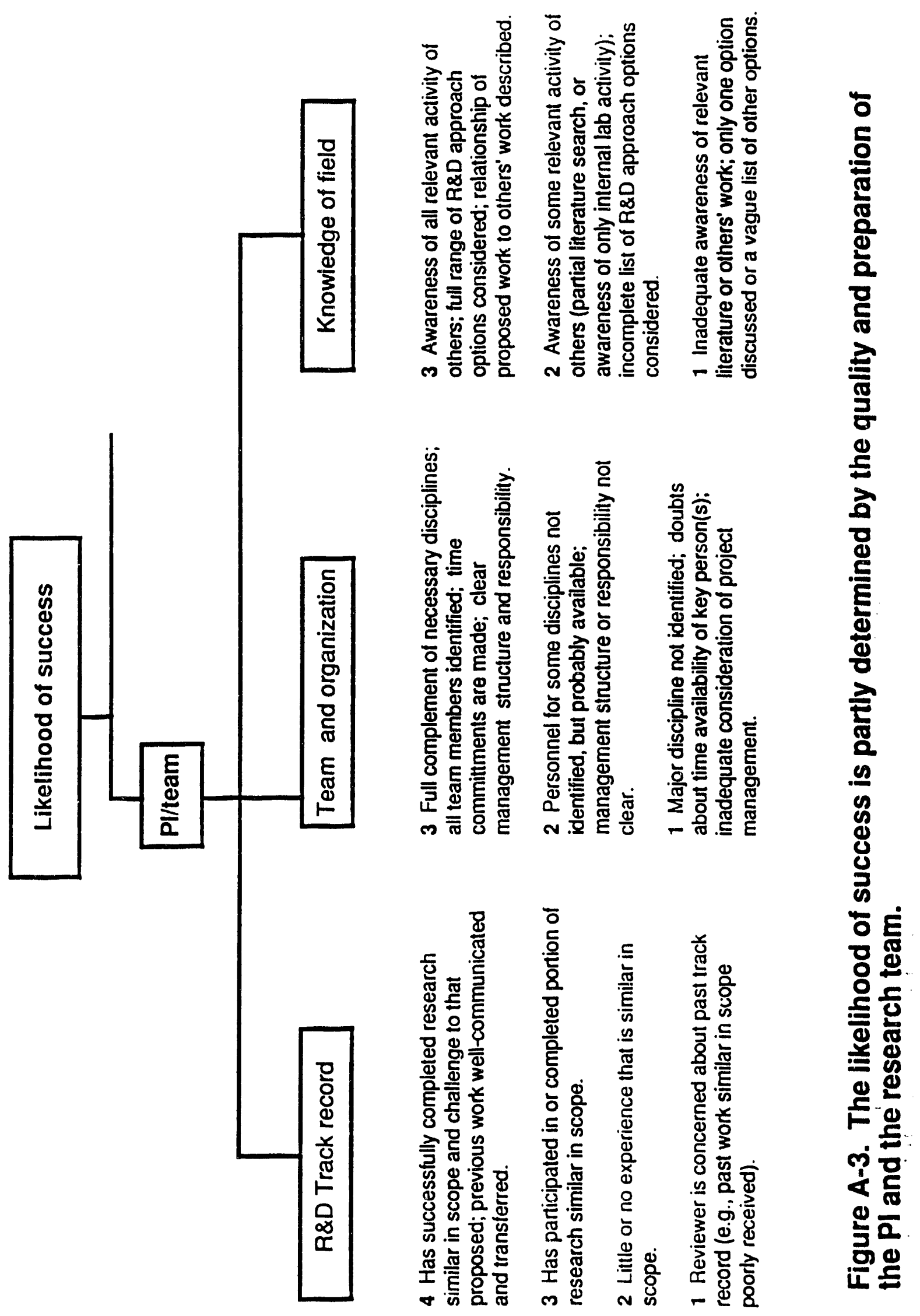




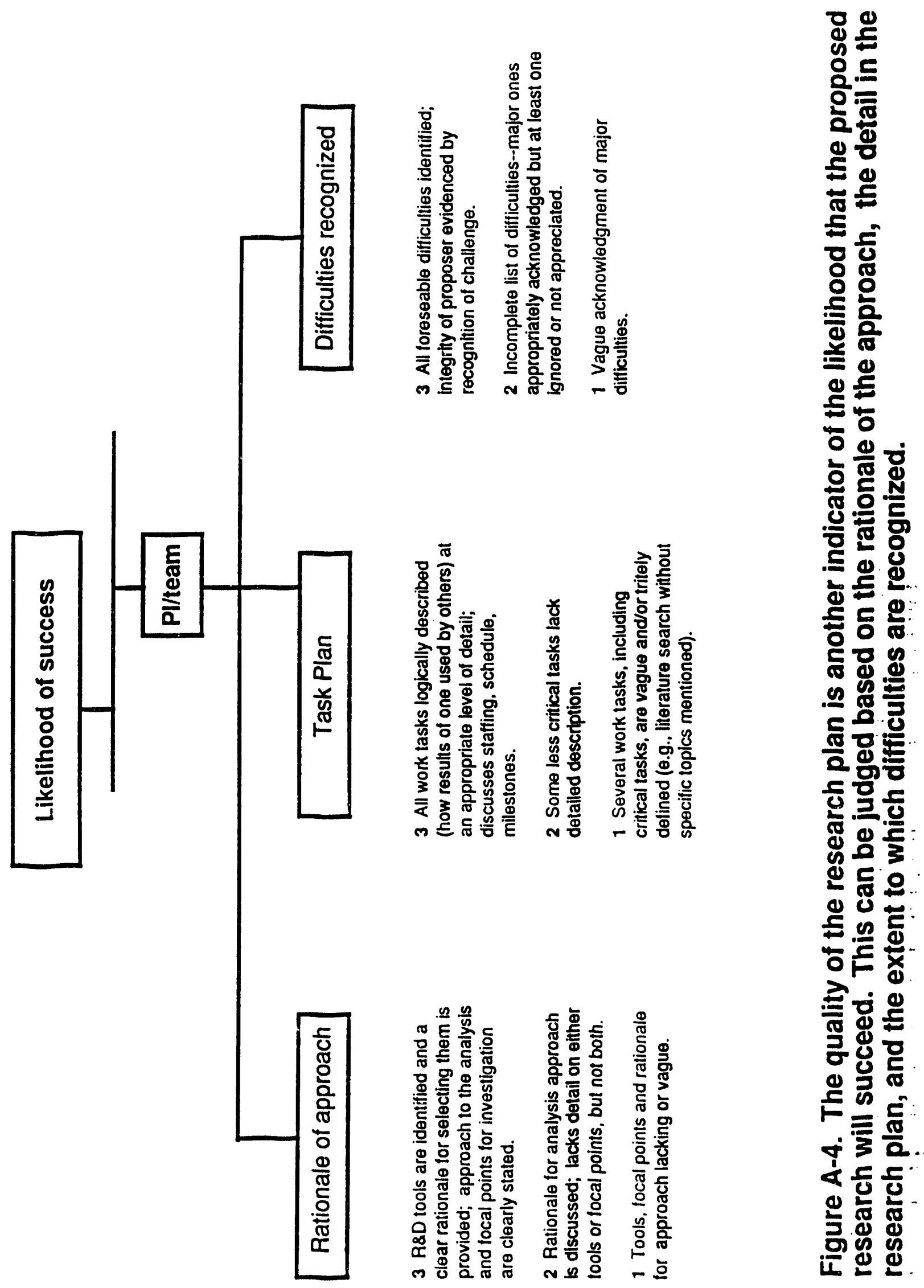



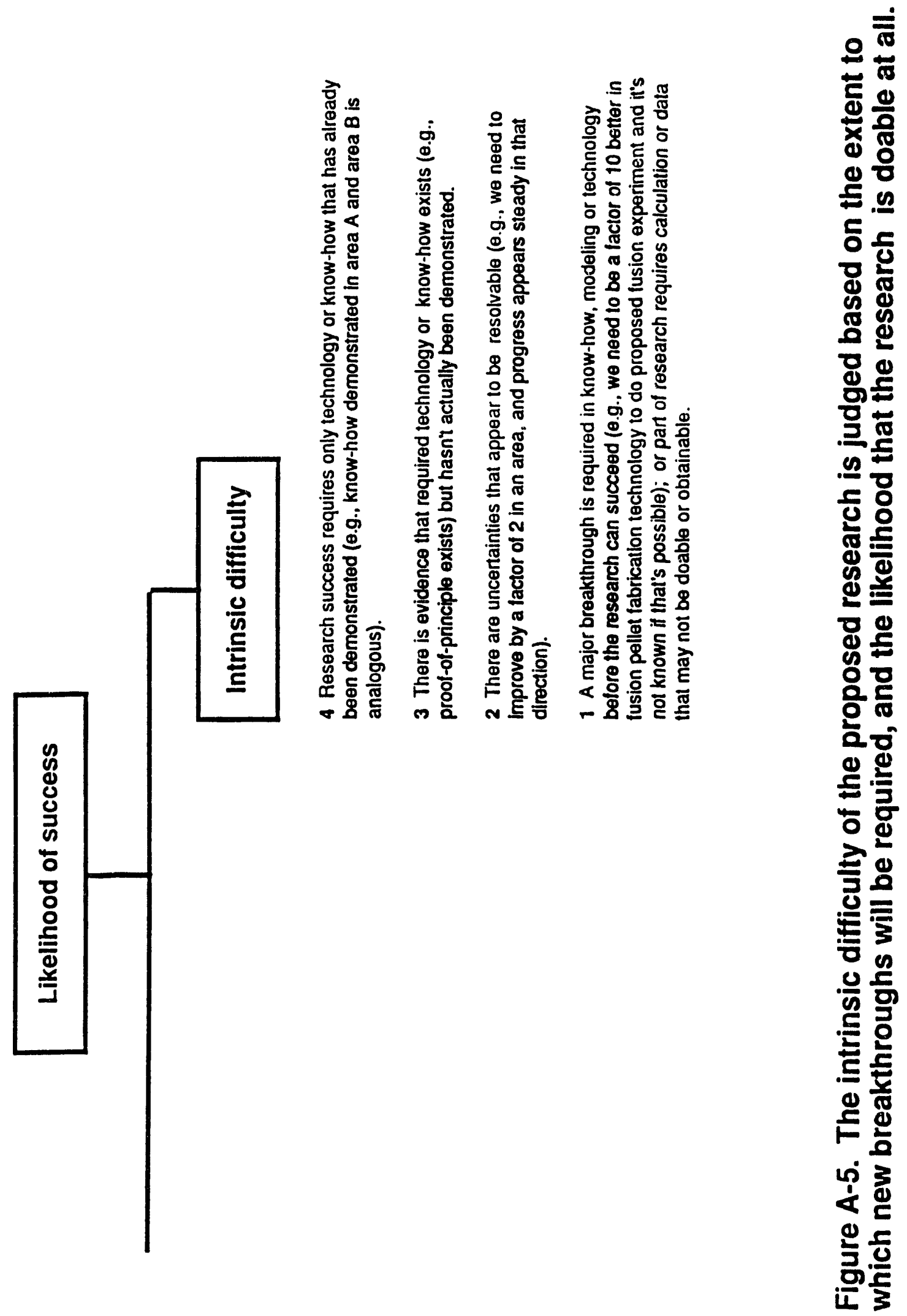


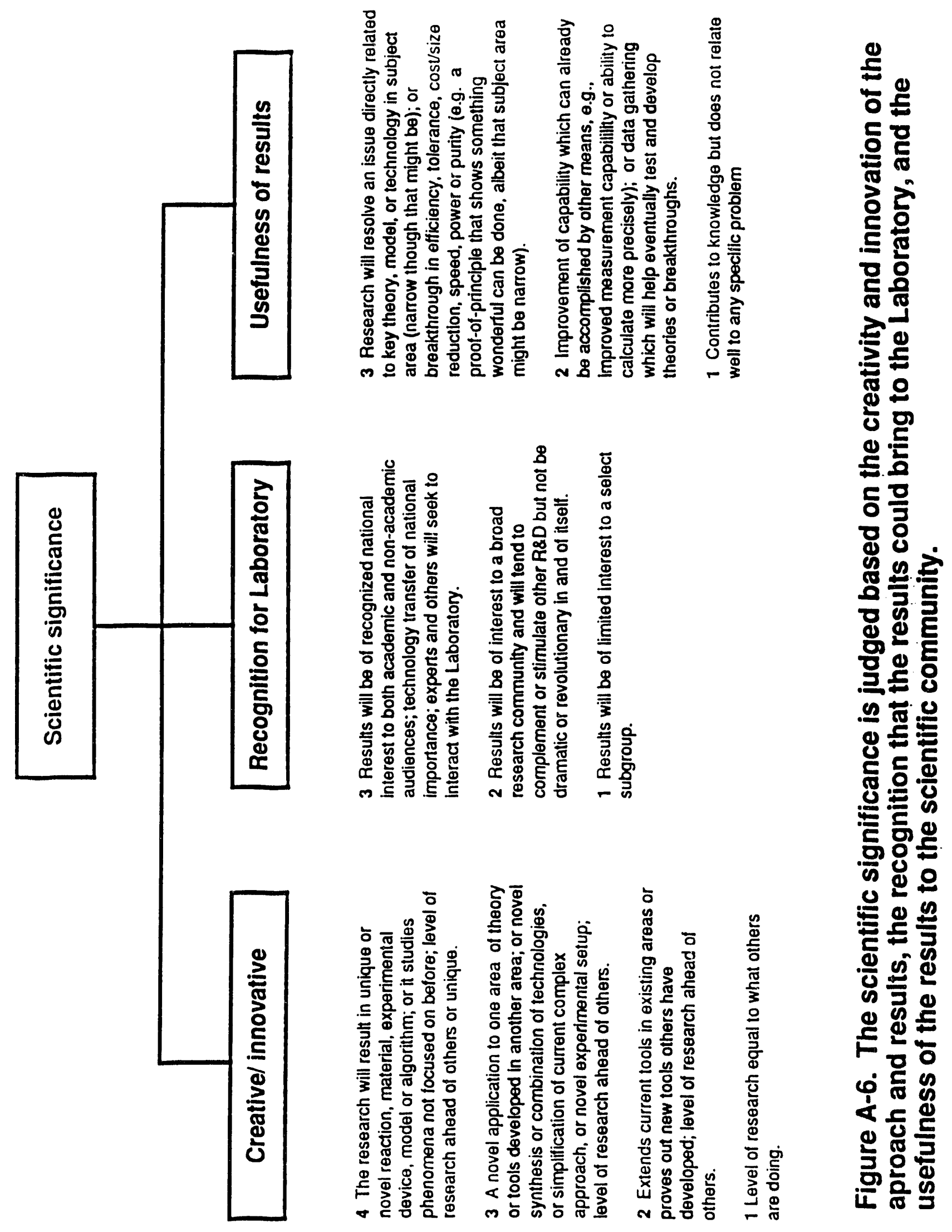




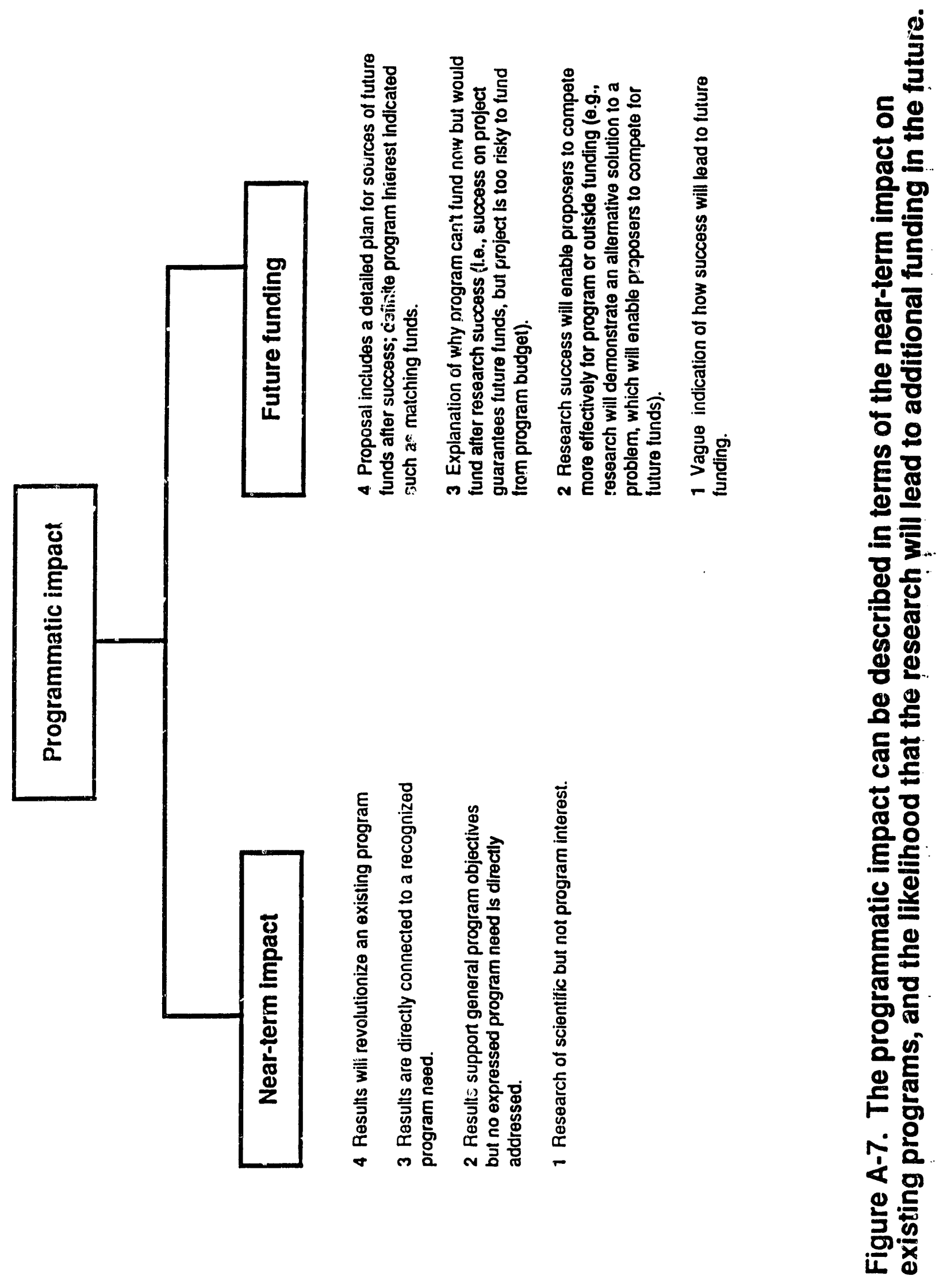




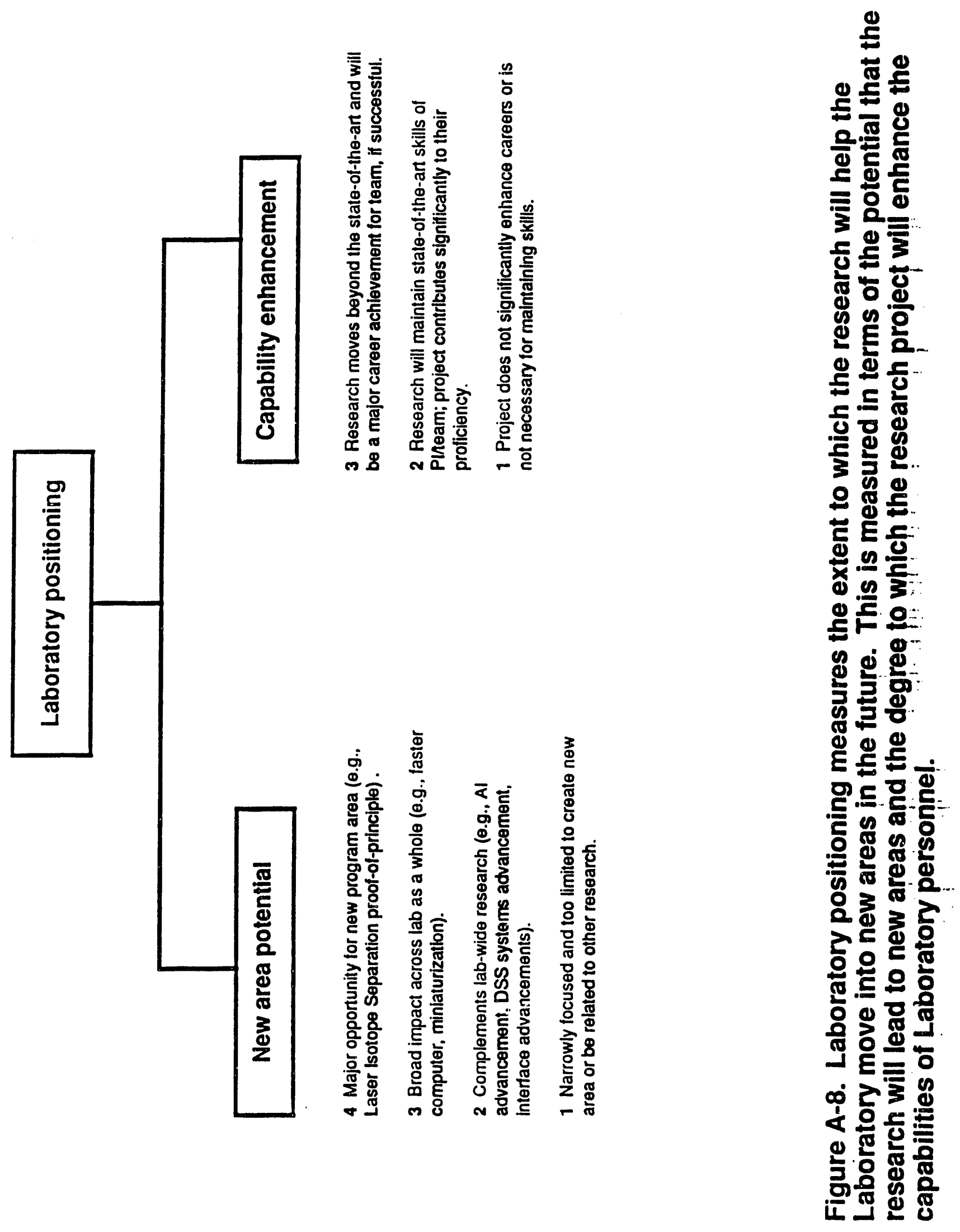




\section{APPENDIX B}

\section{Proposal Ranking (Utility) Function}

\section{Overview}

A ranking or utility function combines the scores for a proposal on the 14 measuring scales (see Appendix A) into one overall score or utility. Important features that a utility function should have are as follows:

- The formulas should be defensible (i.e., formally derived on a theoretically sound basis).

- The formula parameters should be assessable to reflect preference viewpoints in a logical and consistent manner.

The theory and assessment procedures required to determine a utility function with the desired features are discussed in Keeney and Raiffa (1976). In this appendix, we summarize some of the technical details of the utility function form developed for this project.

In mathematical terms, we define a notation for a proposal $X$ which has been assigned levels for each of the 14 measuring scales. We denote these levels by $\left(x_{1}\right.$, $\left.x_{2}, \ldots, x_{14}\right)=\underline{x}$. Similarly, a proposal $Y$ is denoted by $\left(y_{1}, y_{2}, \ldots, y_{14}\right)=\underline{x}$. The question that needs to be answered is whether proposal $X$ is preferred to, indifferent to, or not preferred to proposal $Y$. We would like to make comparisons of such proposals in a systematic, consistent, and theoretically sound manner.

The approach that utility theory takes is to adopt a set of reasonable preference assumptions that can be used to simplify the comparison problem. With these assumptions, it becomes possible for the decision maker or study team to consider simpler comparisons in a consistent manner. Specifically, the simple comparisons never involve more than two measure at a time. A model is then used to establish how 
more complicated comparisons are made. The model ensures that the more complex comparisons are done in a manner consistent with the simpler comparisons and the preference assumptions.

The model referred to in the preceding paragraph is called a multiattribute utility function. It is used to assign a value (symbolically denoted by $u$ ) to each proposal. It does this in such a way so that $u(\underline{X})$ is greater than $u(X)$ if and only if the proposal $X$ is preferred to the proposal $Y$.

The main results of multiattribute utility function theory cover conditions for which a preference model can be expressed in a simple form, and which forms can be reasonably applied to particular problems. The form consists of a function having a number of parameters. These parameters are related to (1) the preferences for different levels of a particular measure and (2) the tradeoffs between pairs of measures indicating their relative importance.

The key aspect of such preference models is that they are derived formally on a theoretically sound basis. While not describing the basis here, the notions involved are essentially those of common sense. For example, one assumption that typifies the kinds of assumptions underlying the model is called transitivity. Transitivity states that if a person prefers $A$ to $B$, and $B$ to $C$, he should prefer $A$ to $C$.

In summary, to address the ranking problem, a mathematical model that makes use of reasonable, well-developed preference assumptions and calibrating procedures is formulated. The model used is now described, along with types of preference input that calibrate the model.

\section{Ranking Function Details}

In our ranking function, the overall utility function is broken up into a combination of simpler functions which can be assessed in a straightforward manner. Specifically, 14 scalar utility functions (SUFs) and 7 multiattribute utility functions (MUFs) have to be assessed. The SUFS deal with the relatively desirability of various levels of each individual measuring scale. (Sometimes preferences are not simply proportional to the level of measure.) The MUFs deal with the tradeoffs between (or relative importance of) different attributes. Note that there is one SUF for each measuring scale, one MUF for each vector attribute and one grand overall MUF. Figure B-1 depicts the hierarchical arrangement of these utility functions.

A SUF is denoted by $u_{i}\left(x_{i}\right)$. Each MUF has the generic form:

$$
u=\frac{\left[\prod_{j=1}^{n}\left[1+k k_{j} u^{j}\right]-1\right]}{k}
$$




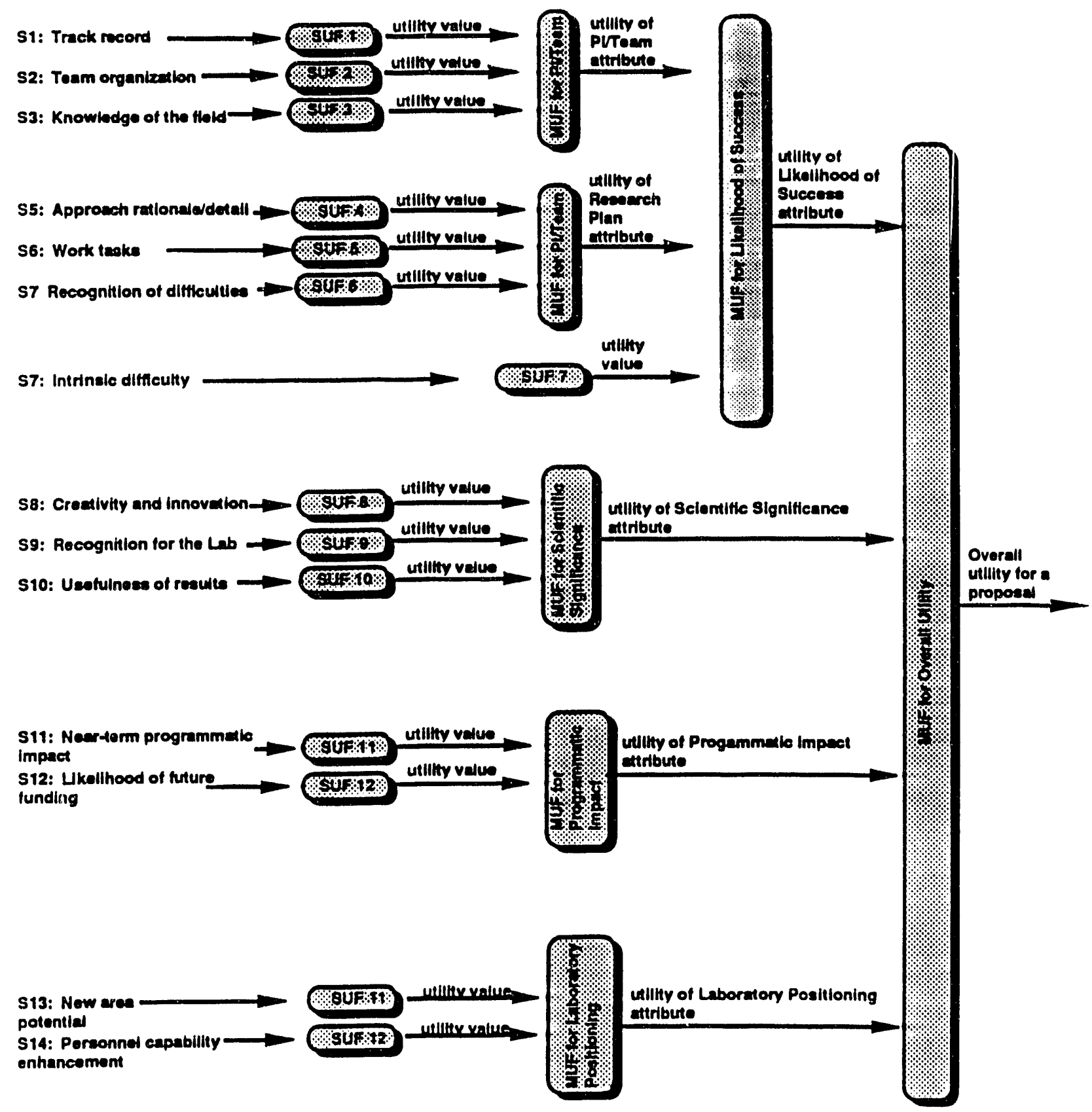

Figure B-1. Hierarchical structure of proposal evaluation utility function. 
where

$$
\begin{aligned}
u & =\text { resulting utility } \\
w & =\text { utility for attribute } j\left(=u_{j}\left(x_{j}\right) \text { when } j \text { is a scalar attribute }\right) \\
k_{1} k_{j} & =\text { Scaling constants specific to each MUF } \\
n & =\text { number of subattributes involved in the calculation }
\end{aligned}
$$

Scaling conventions for these parameters can be used so overall utilities range between 0 and 1 . (The final result is often multiplied by 100 so answers range between 0 and 100.) The multiplicative form above is more flexible than another commonly used model, namely, the additive form. Again, the symbols above refer to parameters in the utility function that reflect preference viewpoints. The hierarchical structure adopted here allows us to rank proposals on subsets of the attributes as well as the complete set. When we rank on a subset of the attributes, we temporarily pretend that the other attributes are not present in the analysis.

The functional forms used are convenient to implement because they involve asking relatively few questions to determine the tradeoff or importance parameters. The forms are general enough, however, to reflect a variety of preferences adequately. With this background, we now describe how the models are calibrated in terms of expressed preferences.

Types of preference questions. There are a few basic types of questions asked of a decision maker (or study team) in calibrating the preference models. Each involves a comparison of hypothetical alternatives that are labeled Alternative $A$ and Alternative $B$. The questioning proceeds until arriving at a point where the two different hypothetical alternatives ( $A$ and $B$ ) are equally preferred by the decision model. A set of these equations is solved to compute the model parameters that will reflect the decision maker's preferences as expressed by comparisons of situations $A$ and $\mathrm{B}$.

Question Type 1: Preference for different levels of a particular measure. In this type of question, only one measure is varied; all other measures are fixed for both $A$ and $B$ (i.e., $A$ and $B$ are identical on all measures except one). For this measure, the following question is posed:

Given the following gamble:

\section{Alternative $A$}

p chance of level 3

$(1-p)$ chance of level 1
Alternative B

versus level 2 for sure

For what $p$ is $A$ indifferent to $B$ ? ( $p$ is the probability of winning).

Questions of this type are asked for all the measures and relative preference values [i.e., the $u_{i}\left(x_{i}\right)$ ] are assigned to reflect the answers of the study team. In the 
example above, the ans:yer to this question determines the value of level 2 relative to levels 1 and 3 for that measure.

Question Type 2: Tradeoffs between two measures. In this type of question, two measures are varied and all other measures are fixed for both $A$ and $B$. The following question is then posed:

Given

$$
\begin{aligned}
& \text { Alternative A } \\
& \left(x_{i}=\right.\text { "good" level, } \\
& x_{i}=\text { "poor" level }
\end{aligned}
$$

\section{Alternative B}

$$
\left(x_{i}=\right.\text { "poor" level, }
$$

or

$$
x_{i}=\text { "good" level) }
$$

Which is the most preferred? "Good" and "poor" are not literally used in the question but rather are used notationally above to stand for a well-defined measure level reflecting a more preferred and less preferred outcome.

Notice that this type of question addresses the issue of tradeoffs (relative importance) directly. In Alternative A, measure " $\mathrm{in}$ is favorable, while " $\mathrm{jm}$ is not. In Alternative $B$, things are reversed. If a person prefers $A$ to $B$, the implication is a willingness to move from the good level to the poor level on measure "j" to improve from the poor level to the good level on measure " $\mathrm{in}$ (i.e., the tradeoff is being expressed). The mathematical techniques for computing the scaling constants and $u_{i}\left(x_{i}\right)$ from the preference information that is input are discussed in Keeney and Raiffe (1976). The important concept is that the calibrated parameters enable the model to reflect consistently the input preferences when it is used to rank proposals.

\section{References}

R. L. Keeney and H. Raiffa, Decision with Multiple Objectives, Wiley, New York, 1976. 


\section{APPENDIX C}

\section{Prototype Software User's Guide}

\section{Overview of the Software Design}

The current software is designed as a database program which (1) maintains information about each proposal and (2) analyses and reports information about the proposals and subsets or groups of proposals.

The current software is not a final product. It is intended to demonstrate the kinds of analyses that can be done using our decision aid approach. Comments about the way this software operates will be useful in guiding the development of a final system.

The program is menu driven. There are help screens and instructions given at many key points, so most of the options are fairly self-explanatory. The following sections describe some of the basic operations that a user might frequently use.

To start the program, type "Foxplus main" at the system prompt. The main menu will come up. It is reproduced here as Fig. C-1. The menu displays eight options for the users. In the sections below, we will discuss four of those options: $P$ (proposal input/update), G (group creation/modification), D (perform dominance relationship calculations), and $R$ (reports).

\section{Input or Update Proposals}

The first operation a user will perform is inputting a set of proposals and their scores on each of the 14 basic attribute scales. From the main menu select "P". This will bring up the Proposal Maintenance menu shown in Fig. C-2. 
From this menu one can browse a proposal (that is look at information about it but not change anything), modify a proposal, or input a new proposal. If the user chooses to either browse or modify a proposal, a screen like that in Fig. C-3 will appear. This shows the current information about the proposal. If the user is browsing, no changes can be made. If the user has selected "modify a proposal" previously, then the data here can be edited. Hitting the "return" key or the $\Uparrow$ or $\downarrow$ cursor keys will move the cursor from field to field.

One note on editing the fields for evaluation criteria or basic attributes: the system will not allow out-of-range levels. For each field, the current maximum permissible level is either 3.0 or 4.0, depending on the field, and the minimum permissible level is 1.0. If the user enters an out-of-range level, the cursor does not move on to the next field when the "return" key is hit. Just enter a permissible value and it will let you proceed.

When the user has finished with the screen in Fig. C-3, the screen in Fig. C-4 comes up. This shows the utility calculations for that proposal. It also shows to which groups this proposal has been assigned.

\section{Perform Dominance Relationship Calculations}

The dominance analysis is a value-free way of comparing proposals which is sometimes useful. We define "dominance" as follows: Proposal A dominates Proposal B if it is better than or equal to B on every attribute, and is strictly better on at least one. If this is the case, then Proposal A is preferred to Proposal B no matter what evaluation function is used. Very good proposals will tend to dominate a large percentage of the other proposals in a group. Conversely, poor proposals will tend to be dominated by many proposals.

The usefulness of the dominance analysis depends on the features of the proposals in a group. It is quite possible that no proposal dominates any other. On the other hand, it can also happen that a subset of proposals tends to dominate all the others. If that is the case, then one can quickly identify the superior proposals in the group without using the utility function.

To execute the dominance analysis calculations, the user selects option $D$ from the main menu (see Fig. C-1). This executes a routine that makes all of the comparisons needed. This takes a long time. There are two phases in the analysis. During the first phase, there is some indication of progress because the screen shows the system counting down through all the proposals. During the second phase, there is nothing on the screen to indicate progress. Since both of these phases takes three or four minutes, it will seem that the machine is frozen. Be patient.

Note that the dominance calculations need to be executed only when new proposals are entered into the database or the attributes of a proposal have been modified. When a proposal is changed, a reminder is displayed on the screen to indicate that the dominance calculations need to be redone. 
The results of the dominance analysis are reported in the ranking reports when one selects the option of displaying percentiles. In this case, the report shows the maximum and minimum percentiles that the proposal can have when compared to the other proposals in the group. The minimum percentile for a given proposal is just the percent of the proposals that it dominates. The maximum percentile is the percent of the proposals that dominate it.

\section{Group Creation/Modification}

The user may wish to define one or more groups of proposals for the analysis. Generally, the user will have at least two groups: one which includes all the proposals that were submitted, and another that includes all those proposals that meet at least some minimum criteria. One might also wish to create a group that contains, say, all the bio-med proposals so that they can be analyzed and ranked together.

A group is created by selecting option $A$ (add a new group definition) from the Group Maintenance screen (see Fig. C-5). The displays that follow will lead the user through the process of creating a group.

One of the steps in creating a group allows the user to define "screening criteria" in selecting proposals for the group. The screening process has two steps. In the first, step all the proposals that fail to meet minimum criteria are screened out. In the second step, the proposals that were screened out are re-examined. If they score favorably on some selected criteria, they are brought back into the group. This twostep prrcess gives the user a great deal of flexibility in setting up the screening proced'ie. In the paragraphs below, we first describe the way the user sets the screening criteria, and we then describe how the system uses the criteria to screen proposals.

The screening criteria are set after selecting option " $\mathrm{S}$ " (screening criteria definition) from the Group Maintenance screen (Fig. C-5). Two displays then come up which allow you to set the "screen-in" and "screen-out" levels. These levels are levels on the basic attribute scales. For example, the screen-out levels are the minimum required levels that a proposal must have in order to be included in the group.

The system uses these screening levels as follows: First there is the "screen-out" step. A proposal is screened out if all of its attribute levels are less than those set in the screen-out criteria. This is intended to screen out proposals that are generally inferior across the board. Then there is the "screen-in" step. This step is applied to those proposals that were screened out. If all its attribute levels are equal to or greater than the level set in the screen-in criteria, then it is brought back into the group. By using combinations of screen-in and screen-out criteria, the user can screen out proposals that are generally inferior, but still bring in proposals which score very high on particular attributes that are of interest to the Committee.

After a group is created, the user may wish to modify it by adding or removing a few selected proposals. This is done by selecting option $M$ (modify an existing group definition). A display will come up which asks which group to modify. Then several 
displays will come up to guide the user through the process of adding or removing proposals.

\section{Reports}

To obtain reports, the user selects option R (reports) from the main menu (Fig. C-1). The reports menu shown in Fig. C-6 will be displayed.

The first six options give reports which rank the proposals according to their utilities on the different supra attributes. An example of the overall ranking report is shown in Fig. C-7.

Option G (group reports) will display a table of information about all the proposals in a group. For each proposal, the table shows all of its attribute levels. The user has the option of displaying either the utility values or the basic attribute levels for proposals in a group.

The other options in the reports menu are somewhat experimental. Option $M$ attempts to show graphically some of the information in the group report. Options $D$ and $B$ show tables of information from the dominance analysis.

\section{A Typical Sequence of Options}

Below, we list a sequence of options that a user can perform with the prototype software. This sequence relates to the four basic steps in Fig. 1 of the main report for evaluating and ranking proposals. The software main menu and submenu option letters are listed in parentheses for each step in the sequence.

1. Describe proposals (or retrieve proposal data)
$(P, A)$
Input/update and add new proposals
$(\mathrm{P}, \mathrm{B})$
Input/update and browse any existing proposal

2. Screen out low scoring proposals
$(G, S)$
Screening criteria definition
$(G, A)$
Add a new group definition (subset absent in low-scoring proposals)

3. Rank proposals

Utility function parameters (set after preferences are assessed) and automatic calculation of proposal utilities. 
$(R, O) \quad$ Report (showing proposals rank-ordered by overall utility and cumulative costs)

4. Display and examine best proposals

(R, S, etc..) Report (showing proposals rank-ordered by scientific significance utility, etc.)

(G)

Group report (detailed report of each proposal in any selected subset or group) 


$$
\begin{aligned}
& \text { LAWFENCE L.: VEFIMORE LABOFATORY } \\
& \text { FROFOSAL MANAGEMENT SYSTEM }
\end{aligned}
$$

Salace ail Uation:

F) Froposai Inout/Undace

i) Group Lreatzon/mooz d catzon

(i) Fiepores

D) Fertorm Dominance fielationsnip Calculations

U) Utility Function Farametors

i) E::it to dBASE Dot Firompt

i) Ro-initialize Group Calc/Relationsnip files

E) End Frocessing

Enter: $F$

Figure C-1. Main menu.

LAWFEHCE LI'VERMORE LAEOFATOFYY FROFOSAL EUALUATION G'ISTEM FROFOSAL MAINTENANCE

Teiki: r. in sotion:

$\therefore$ Lrowse ill E:a lstina Frooosal

(1) llods1: ar, E:: lstina Frodosal

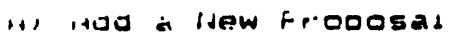

(i) L'Elete a Froposal

(i) Fie-calculace ill frodosal Utillt $\because$ Values

E) E.:1t this Screen

Enter:

\section{Figure C-2. Proposal input/update menu.}




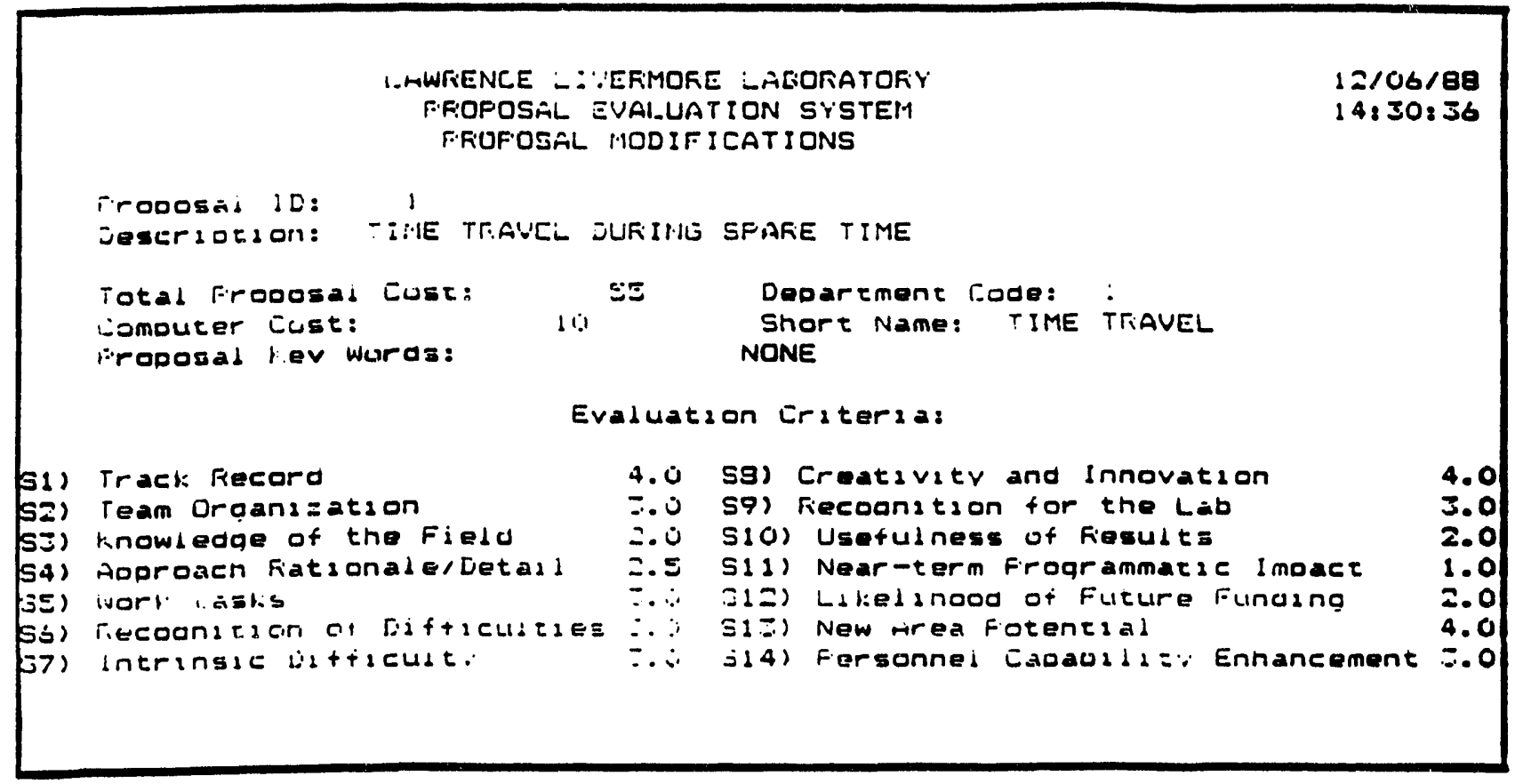

\section{Figure C-3. Proposal input screen.}

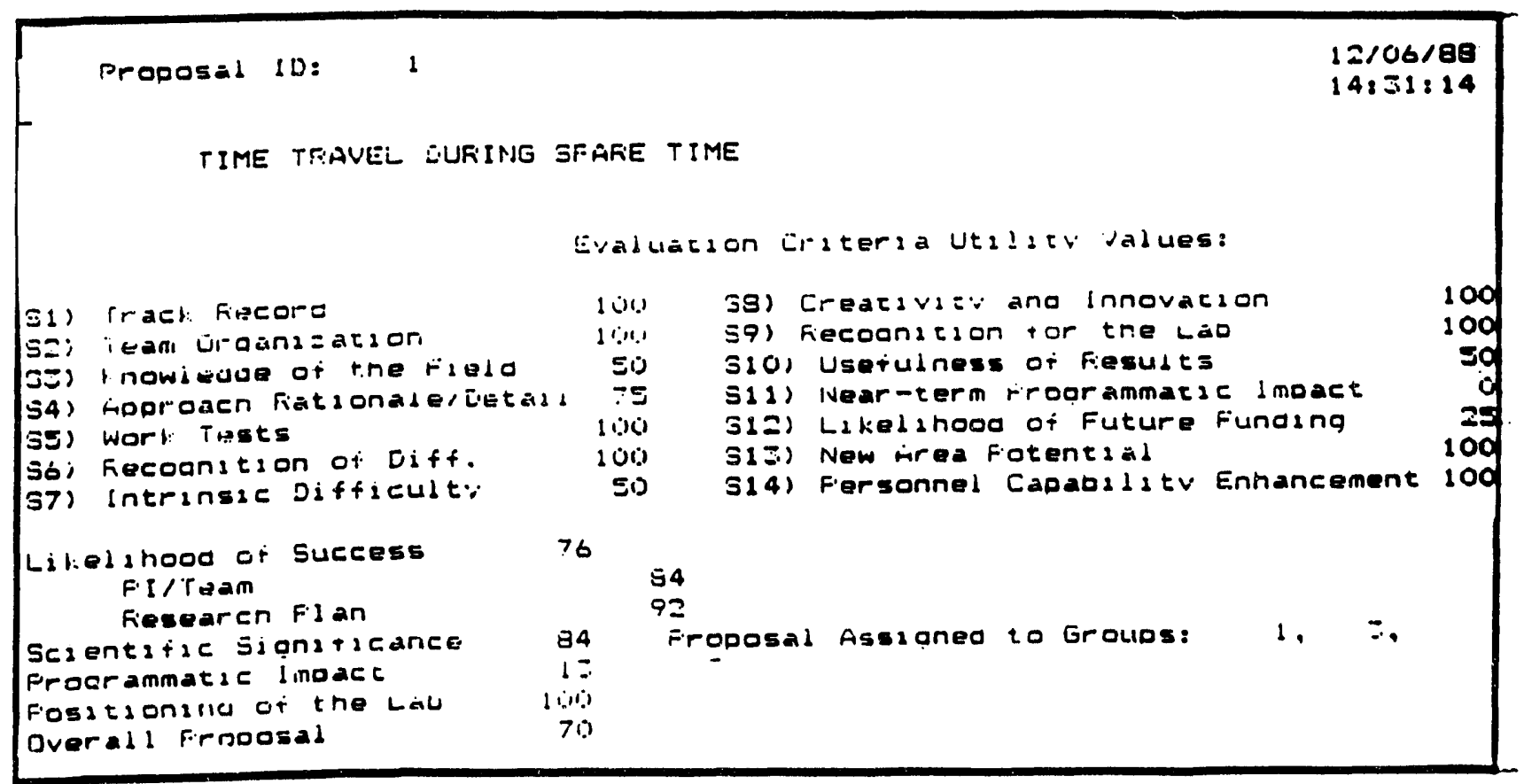

Figure C-4. Proposal browse output, second display. 
LAWRENCE : :U'ERMORE LAEDRATORY

PROPOSAL EUALUATION SISTEM

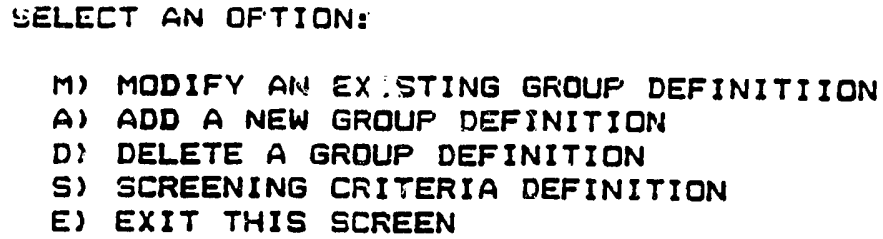

D) DELETE A GROUP DEFINITION

S) SCREENING CRITERIA DEFINITION

E) EXIT THIS SCREEN

ENTER: $E$

\title{
Figure G-5. Group creation/modification menu.
}

\author{
LAWRENCE LIVERMORE LABORATORYY \\ $12 / 06 / 80$ \\ F.ROFOSAL MANAGEMENT SYSTEM

Select an Dotzon:

i) Fil Team Fianked Listing

(r) Fiesearch Flan Fankeo Listino

E: Scientitic Sianiticance Ranked $1: s=1 n g$

I) Frooramatic lmoact Fianked listino

L) Fositioning of the Lab Ranked listing

a) Overall Value fianked listing

G) Groub fieports

D) fieport proposals dominating a selected proposal

E) Fi port proposals domanated by a selacted proposal

M) Fircoosal Matra:: Demo

E) E::it to Main Menu

ENTEF: E

i Figure C-6. Report menu. 


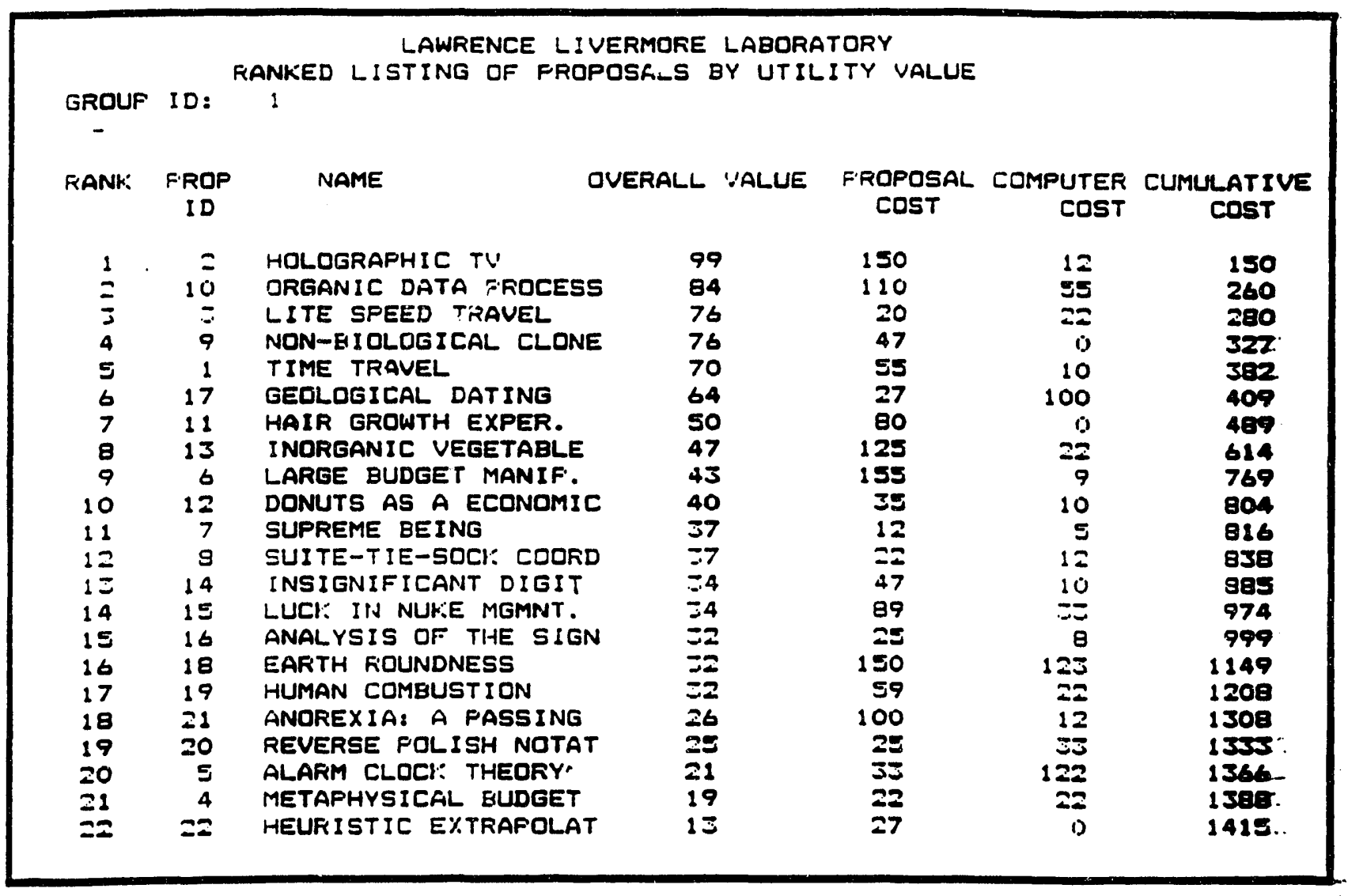

Figure C-7. Illustrative overall ranked listing. 


\section{APPENDIX D}

March Proposal Memo to the IR\&D Committee 
interdepartmental letterhead

Mail StationL. 195

Ext: $\quad 2-8467$

March 15, 1988

SRG880319

TO: Institutional Research and Development Research Committee

FROM: $\quad$ R. A. Al-Ayat

SUBJECT: Developing a Decision Aid for Evaluating and Selecting Re्य Proposais

This memo describes possible assistance to the IR\&D Committee regarding the development of a systematic framework for evaluating and selecting R\&D proposals. We begin with a discussion of elements of the problem facing the IR\&D Research Committee. Then we briefly describe areas where we can provide assistance. Next, we describe possible tasks which can be conducted sequentially, depending on funding and the Committee's needs.

INTRODUCTION

The IR\&D Research Committee faces the challenging task of selecting R\&D proposals for funding. One can view the R\&D selection as a two-step process. First, individual proposals are evaluated for their scientific merit and potential laboratory impact, if successful. At this point, many proposals are eliminated from consideration. Second, the remaining proposals (short list) are ranked, and a group or portfolio of proposals, consistent with the funding constraint, is selected. These steps require evaluation criteria and an approach to select a portfolio that addresses the goals of the Committee and the Laboratory.

Members of the Committee use various approaches for evaluating proposals and for quantifying their feelings about the quality and likelihood of success of a given proposal. However, ranking of proposals and choosing a portfolio, which is done in committee meetings, presents an additional challenge to the committee members. Time and funding constraints give rise to many concerns including:

(a) good proposals may not be given adequate recognition or be effectively advocated during the Committee's deliberation;

(b) a chosen portfolio may not necessarily maximize the return to the Laboratory in terms of innovative results, scientific recognition, future funding (leverage), and new areas of work;

\section{Universtry of California}

\section{UL Lawrence Livermore National Laboratory}


(c) the chosen portfolio may not be sufficiently "bzlanced." (For example, many proposals may be funded in a given area than are necessary to stimulate research. In this case, funding would not be available to promote additional areas of work that could bring added recognition to the Laboratory.)

The goal of the tasks described below is to assist the Committee by developing a systematic framework for evaluating and ranking submitted proposals and for choosing a balanced portfolio that meets the overall goals of the IR\&D. Research Committee. By working with Committee members, we hope to provide an approach that synthesizes the Committee's goals, streamiines the review process, and reduces the time required to reach consensus and select an R\&D portfolio to fund.

\section{Proposed Tasks and Deliverables}

We have divided the proposed effort into 6 tasks. These can be conducted in a sequential manner depending on the availability of funds and the committee's needs.

Task 1 Develop a systematic procedure for evaluating the merit of individual proposals. In this task we will work closely with Committee members to identify the factors that should be considered in evaluating the significance of a research proposal and the likelihood of research success. We will develop a checklist of measures that will help Committee members summarize the key factors for each proposal. Instead of simple "1-5" scales for judging proposal quality, these measures will help provide explicit descriptions of how well any given proposal addresses various technical concerns. The checklist formalism could help ensure that meritorious proposals are not over looked.

Task 2 Develop a utility function to help rank order proposals on technical merit. The utility function synthesizes the scores proposals receive on the measures from Task 1 and produces an overall figure of merit for each proposal. Preference assessments obtained through interviews with the Committee control how proposals are ranked by the utility function. Assessment techniques make use of systematic checks for logic, consistency, and agreement of ranking with committee members' preferences.

Task 3 Develop software to store data about, and display rankings and key features of proposals. The software will help the Committee to compare systematically the strengths and weaknesses of different proposals side by side, using the checklists developed in Task 1. The software will also display characteristics of any group of proposals, such as the number in various discipline areas, cost ranges, and mission areas. Software implementation will feature concise graphical displays (e.g., bar charts) and a convenient interface (e.g., Excel on a Macintosh).

Task 4 Develop a checklist for describing the global characteristics of a portfolio of proposals. This checklist will help identify desired characteristics of a portfolio of proposals. Identifying these 
characteristics of a portfolio of proposals. Identifying these characteristics will make it possible to compare alternative portfolios and identify those that are not balanced or may not achieve the highest return to the Laboratory.

Task 5 Develop a utility function for a portfolio of proposals. The utflity function helps compare portfolios on technical merits. Committee members' preferences obtained through interviews will control how portfolios are compared. A key trade-off involved is illustrated heuristically by the following: "Is it preferable to fund five higher quality proposals in a specific area or eight proposals of slightly lesser quality in a broader range of areas, given that both sets can be done for the same budget?" Currently, proposals are selected from the short list starting with the top-ranked proposal until funds are exhausted. This may overlook alternate portfolios that would result in a greater overall benefit to the Laboratory.

Task 6 Develop software to heip identify an optimal portfolio. This software would use the utility function developed in Task 5 to suggest a portfolio maximizing technical quality and return to the Laboratory while satisfying balance constraints. The software would allow the Committee to explore how the suggested portfolio would look if funding were changed, various proposals were mandated in or out, or percentages or numbers of projects in different areas were required or relaxed.

Tasks 1-3 provide basic steps for systematically describing, displaying, and rank-ordering of proposals. This in itself should prove valuable to the Committee and would be the basis for Tasks 4-6.

Tasks 4-6 provide a tool for portfolio selection that is driven by preference input obtained from members of the Committee. The tool's main use is to evaluate alternative portfolios, perform sensitivity analysis to various judgments, and suggest portfolios for the Committee's consideration. An advantage of these tools is that ranking and portfolio results can be systematically traced to proposal scores on evaluation criteria and on the Committee's preference inputs.

\section{Costs}

For FY88, we propose doing Tasks 1, 2, and either Task 3 or Task 4. These tasks can be accomplished at $\$ 25 \mathrm{~K}$ to $\$ 35 \mathrm{~K}$. The remaining tasks could be pursued at a later date, depending on the Committee's needs.

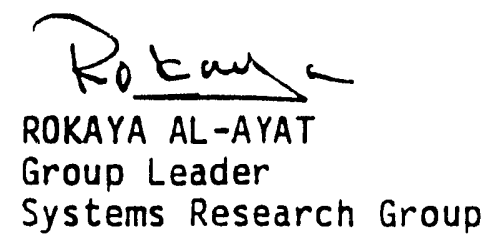



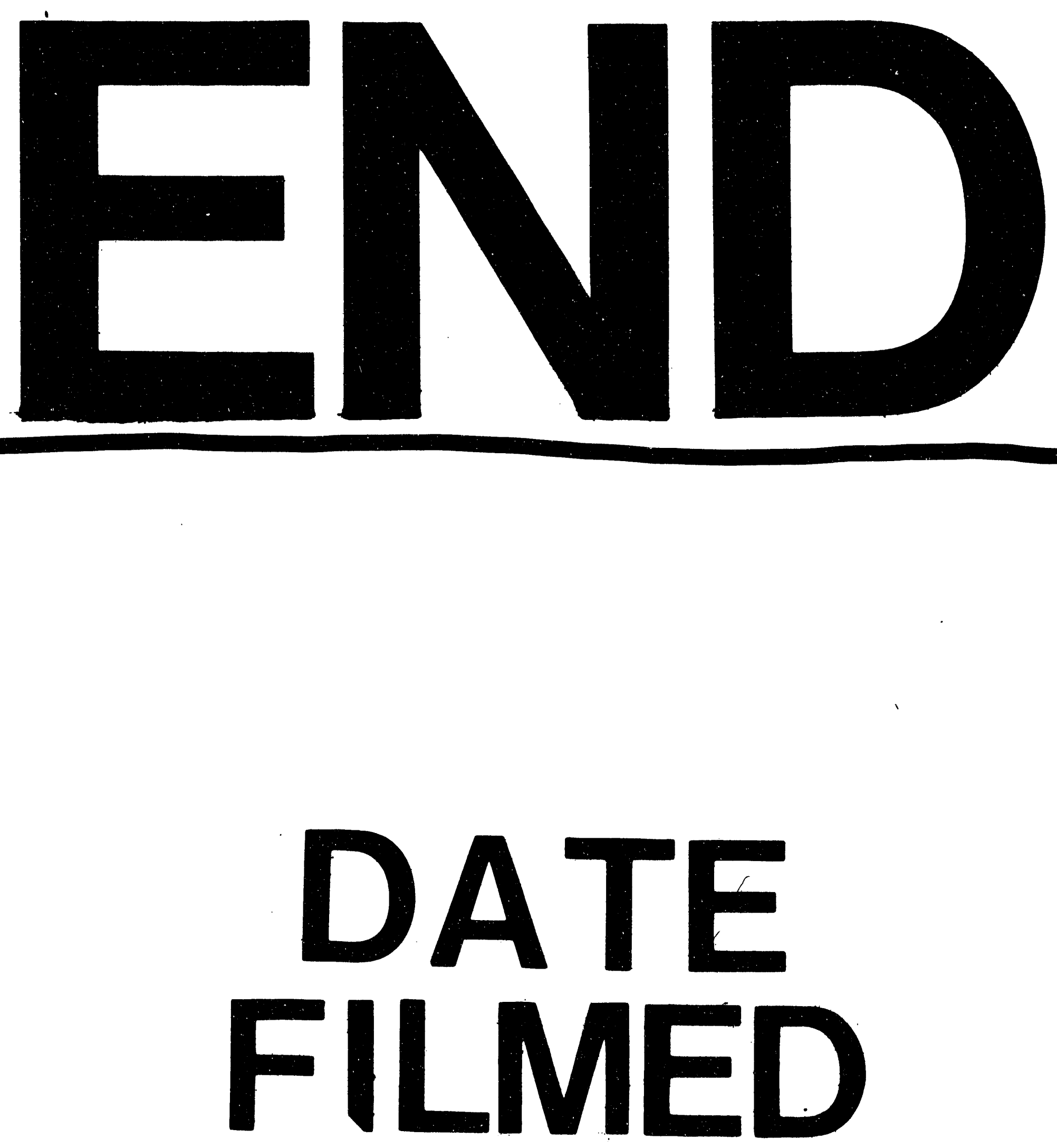

$9 / 13 / 93$ 
